九州大学学術情報リポジトリ

Kyushu University Institutional Repository

\title{
CONDITIONAL PROBABILITY DENSITY AND REGRESSION FUNCTION ESTIMATIONS WITH TRANSFORMATION OF DATA
}

Mor iyama, Taku

Dept. of Management of Social Systems and Civil Eng., Tottori University

Maesono, Yoshihiko

Department of Mathematics, Chuo University

https://doi.org/10.5109/2558879

出版情報: Bulletin of informatics and cybernetics. 52 (1)，pp.1-25，2020. Research Association of Statistical Sciences

バージョン :

権利関係 : 
CONDITIONAL PROBABILITY DENSITY AND REGRESSION FUNCTION ESTIMATIONS WITH TRANSFORMATION OF DATA

by

Taku Moriyama and Yoshihiko Maesono

Reprinted from the Bulletin of Informatics and Cybernetics

Research Association of Statistical Sciences, Vol.52

FUKUOKA, JAPAN

2020 


\title{
CONDITIONAL PROBABILITY DENSITY AND REGRESSION FUNCTION ESTIMATIONS WITH TRANSFORMATION OF DATA
}

\author{
By
}

Taku MoriYama* and Yoshihiko Maesono ${ }^{\dagger}$

\begin{abstract}
This study extends 'empirical transformation' in kernel smoothing to conditional probability density and regression function estimations. We propose nonparametric conditional probability density and regression function estimators, which are based on an extension of Ćwik and Mielniczuk (1989)'s method. We derived asymptotic properties of the proposed estimators and conducted a simulation that showed mean squared errors of conditional probability density and regression function estimators.
\end{abstract}

Key Words and Phrases: conditional probability density, mean squared error, nonparametric estimation, regression function.

Let $\left(X_{1}, Y_{1}\right), \cdots,\left(X_{n}, Y_{n}\right)$ be $n$ pairs of independent random variables (r.v.s.) with the continuous joint density function $f(x, y)$ and $g(x)$ be the marginal density of $X$. Throughout the paper, we assume that the support of $f$ covers $\mathbb{R}^{2}$ to avoid what we call the 'boundary bias problem'. The conditional probability density of $Y$ given $X=x_{0}$ (fixed) is given by

$$
f_{Y \mid x_{0}}(y):=f_{Y}\left(y \mid X=x_{0}\right)=\frac{f\left(x_{0}, y\right)}{g\left(x_{0}\right)} .
$$

Conditional density estimation provides not only its mean but also its mode, multimodality, skewness, quantile points and so on. Rosenblatt (1969) first considered nonparametric kernel-type estimation. The estimator at $Y=y_{0}$ (fixed) is

$$
\widehat{f}_{Y \mid x_{0}}\left(y_{0}\right)=\frac{\widehat{f}\left(x_{0}, y_{0}\right)}{\widehat{g}\left(x_{0}\right)}
$$

where $\widehat{f}$ and $\widehat{g}$ are the 'naïve' kernel density estimators:

$$
\widehat{f}\left(x_{0}, y_{0}\right)=\frac{1}{n h_{1} h_{2}} \sum_{i=1}^{n} K\left(\frac{x_{0}-X_{i}}{h_{1}}\right) K\left(\frac{y_{0}-Y_{i}}{h_{2}}\right)
$$

\footnotetext{
* Dept. of Management of Social Systems and Civil Eng., Tottori University, 4-101 Koyama-Minami Tottori 680-8550 Japan. tel +81-857-31-5335 moriyama@tottori-u.ac.jp

$\dagger$ Department of Mathematics, Chuo University, 1-13-27 Kasuga, Bunkyo-ku Tokyo 112-8551 Japan. tel +81-03-3817-1762 maesono@math.chuo-u.ac.jp
} 
and

$$
\widehat{g}\left(x_{0}\right)=\frac{1}{n h_{3}} \sum_{i=1}^{n} K\left(\frac{x_{0}-X_{i}}{h_{3}}\right),
$$

where $K$ is a symmetric probability density function and $h_{j}(j=1,2,3)$ are the bandwidths. We assume that all the bandwidths in the estimators are the same, i.e., $h_{j}=h$ $(j=1,2,3)$, where $h \rightarrow 0$ and $n h \rightarrow \infty$ as $n \rightarrow \infty$. Let us use the following notation,

$$
A_{i, j}:=\int K^{i}(u) u^{j} \mathrm{~d} u
$$

We also assume that all $A_{1, j}$ for $j=0, \cdots, 4$ and $A_{2, k}$ for $k=0,1$ are bounded.

Hyndman et al. (1996) investigated the mean squared error of the kernel-type conditional density estimator and proposed an improved estimator.

Bashtannyk and Hyndman (2001) discussed methods of choosing the bandwidth of the conditional density estimator from the view of asymptotic results. Hall et. al. (2004) proved that a cross-validation method is quite effective for automatically shrinking and removing irrelevant explanatory components in multivariate settings. Hall et. al. (1999) introduced and investigated local logistic and adjusted the Nadaraya-Watson method for nonparametric conditional density estimation. Otneim and Tjøstheim (2017) proposed a method for tackling the curse of dimensionality in nonparametric settings using locally Gaussian approximations.

As pointed out by Faugeras (2009), the ratio-type estimator $\widehat{f}_{Y \mid x_{0}}$ can be numerically unstable when the denominator $\widehat{g}\left(x_{0}\right)$ is close to zero. Faugeras (2009) proposed a 'product-shaped estimator':

$$
\widehat{f}_{Y \mid x_{0}}^{\dagger}\left(y_{0}\right)=\widehat{c}\left(G_{n}\left(x_{0}\right), L_{n}\left(y_{0}\right)\right) \widehat{\ell}\left(y_{0}\right)
$$

where $\widehat{c}$ is a copula density estimator and $G_{n}, L_{n}$ are the empirical distribution functions of the marginal distribution functions of $X$ and $Y$, respectively. $\widehat{\ell}$ is the kernel density estimator of the r.v. $Y$. The idea is based on the property of copula $C$; for any bivariate cumulative distribution function (c.d.f.) $F$, with marginal c.d.f. $G$ of $X$ and $L$ of $Y$, there exists some function $C:[0,1]^{2} \rightarrow[0,1]$. Copula $C$ is called the copula function, which satisfies

$$
F\left(x_{0}, y_{0}\right)=C\left(G\left(x_{0}\right), L\left(y_{0}\right)\right)
$$

(known as Sklar's theorem). If $G$ and $L$ are continuous, this representation is unique. Copula $C$ is itself a c.d.f. on $[0,1]^{2}$ with uniform marginal distributions. By differentiating the formula, we have the following representation:

$$
f\left(x_{0}, y_{0}\right)=c\left(G\left(x_{0}\right), L\left(y_{0}\right)\right) g\left(x_{0}\right) \ell\left(y_{0}\right),
$$

where $c$ is the copula density function and $\ell$ is the marginal density of $Y$. The above estimator $\widehat{f}_{Y \mid x_{0}}^{\dagger}$ comes from this relation. Faugeras (2009) investigated some asymptotic properties of this estimator.

By extending the idea of Ćwik and Mielniczuk (1989), we propose the following conditional density estimator:

$$
\widehat{f}_{Y \mid x_{0}}^{\star}\left(y_{0}\right):=\frac{1}{n h^{2}} \sum_{i=1}^{n} K\left(\frac{Y_{i}-y_{0}}{h}\right) K\left(\frac{G_{n}\left(X_{i}\right)-G_{n}\left(x_{0}\right)}{h}\right) .
$$


The proposed estimator $\widehat{f}_{Y \mid x_{0}}^{\star}$ can be seen as an estimator with 'empirical transformation' $\left(X \mapsto G_{n}(X)\right)$ (see Ruppert and Cline (1994)). Since $\widehat{f}_{Y \mid x_{0}}^{\star}$ is not a ratio, we also expect it to have less variance, as Faugeras (2009) did.

Since both Faugeras (2009)'s estimator $\widehat{f}_{Y \mid x}^{\dagger}$ and our $\widehat{f}_{Y \mid x}^{\star}$ use the empirical distribution function $G_{n}$, they are discontinuous functions as $x$. Let us replace $G_{n}$ with the kernel-type estimator $\widehat{G}$. Then, we propose the following conditional density estimator:

$$
\widehat{f}_{Y \mid x_{0}}^{\diamond}\left(y_{0}\right):=\frac{1}{n h^{2}} \sum_{i=1}^{n} K\left(\frac{Y_{i}-y_{0}}{h}\right) K\left(\frac{\widehat{G}\left(X_{i}\right)-\widehat{G}\left(x_{0}\right)}{h}\right) .
$$

We assume that the bandwidth of $\widehat{G}$ coincides with $h$, which is used in this estimator $\widehat{f}_{Y \mid x_{0}}^{\diamond}$. Though the convergence rate of $\widehat{f}_{Y \mid x_{0}}^{\diamond}$ is not improved, as shown later, the kernel estimator $\widehat{f}_{Y \mid x}^{\diamond}\left(y_{0}\right)$ is a smooth function of $x$ unlike $\widehat{f}_{Y \mid x}^{\star}\left(y_{0}\right)$. The asymptotic mean squared errors $(A M S E s)$ of $\widehat{f}_{Y \mid x_{0}}^{\star}$ and $\widehat{f}_{Y \mid x_{0}}^{\diamond}$ are given in the following theorems.

Theorem 1 Let us assume that (i) $f$ and $g$ are three-times differentiable at $\left(x_{0}, y_{0}\right)$ and at $x_{0}$ respectively, (ii) $f^{(i, 0)}\left(x_{0}, y_{0}\right), g^{(i)}\left(x_{0}\right)$ and $f^{(0, j)}\left(x_{0}, y_{0}\right)$ are bounded for $i=$ $0,1,2,3$ and $j=1,2,3$, (iii) $K$ is three-times continuously differentiable, (iv) is the support of $K$ is given by $[-d, d]$ for some positive constant $d>0$, and $(\mathrm{v}) \sup _{v}\left|K^{(3)}(v)\right|$ is bounded. Then, the AMSE of $\widehat{f}_{Y \mid x_{0}}^{\star}\left(y_{0}\right)$ is given by

$$
\begin{aligned}
& E\left[\widehat{f}_{Y \mid x_{0}}^{\star}\left(y_{0}\right)-f_{Y \mid x_{0}}\left(y_{0}\right)\right]^{2} \\
= & \frac{h^{4} A_{1,2}^{2}}{4}\left[f^{(0,2)}\left(x_{0}, y_{0}\right)-f_{Y \mid x_{0}}\left(y_{0}\right) \frac{g\left(x_{0}\right) g^{\prime \prime}\left(x_{0}\right)-3\left(g^{\prime}\left(x_{0}\right)\right)^{2}}{g^{4}\left(x_{0}\right)}\right. \\
& \left.\quad+\frac{1}{g^{4}\left(x_{0}\right)}\left(3 g^{\prime}\left(x_{0}\right) f^{(1,0)}\left(x_{0}, y_{0}\right)-g\left(x_{0}\right) f^{(2,0)}\left(x_{0}, y_{0}\right)\right)\right]^{2} \\
& +\frac{A_{2,0}^{2}}{n h^{2}} f_{Y \mid x_{0}}\left(y_{0}\right)+O\left(h^{6}\right)+O\left(\frac{1}{n h}\right),
\end{aligned}
$$

where

$$
f^{(i, j)}\left(x_{0}, y_{0}\right)=\left.\left(\frac{\partial}{\partial w}\right)^{i}\left(\frac{\partial}{\partial z}\right)^{j} f(w, z)\right|_{w=x_{0}, z=y_{0}} .
$$

Proof: See the appendix. 
Theorem 2 Under the assumptions of Theorem 1, the AMSE of $\widehat{f}_{Y \mid x_{0}}^{\diamond}\left(y_{0}\right)$ is given by

$$
\begin{aligned}
& E\left[\widehat{f}_{Y \mid x_{0}}^{\diamond}\left(y_{0}\right)-f_{Y \mid x_{0}}\left(y_{0}\right)\right]^{2} \\
= & \frac{h^{4} A_{1,2}^{2}}{4}\left[\frac{2 f\left(x_{0}, y_{0}\right) g^{\prime \prime}\left(x_{0}\right)}{g^{\prime}\left(x_{0}\right)}+f^{(0,2)}\left(x_{0}, y_{0}\right)-f_{Y \mid x_{0}}\left(y_{0}\right) \frac{g\left(x_{0}\right) g^{\prime \prime}\left(x_{0}\right)-3\left(g^{\prime}\left(x_{0}\right)\right)^{2}}{g^{4}\left(x_{0}\right)}\right. \\
& \left.\quad+\frac{1}{g^{4}\left(x_{0}\right)}\left(3 g^{\prime}\left(x_{0}\right) f^{(1,0)}\left(x_{0}, y_{0}\right)-g\left(x_{0}\right) f^{(2,0)}\left(x_{0}, y_{0}\right)\right)\right]^{2} \\
& +\frac{A_{2,0}^{2}}{n h^{2}} f_{Y \mid x_{0}}\left(y_{0}\right)+O\left(h^{6}\right)+O\left(\frac{1}{n h}\right) .
\end{aligned}
$$

Proof: See the appendix.

Remark 1 As seen above, the difference between the AMSEs of $\widehat{f}_{Y \mid x_{0}}^{\star}$ and $\widehat{f}_{Y \mid x_{0}}^{\diamond}$ is whether the first term in the bias terms exists. Under some regularity conditions, the $A M S E$ of $\widehat{f}_{Y \mid x_{0}}\left(y_{0}\right)$ is given by

$$
\begin{aligned}
& E\left[\widehat{f}_{Y \mid x_{0}}\left(y_{0}\right)-f_{Y \mid x_{0}}\left(y_{0}\right)\right]^{2} \\
= & \frac{h^{4} A_{1,2}^{2}}{4} \frac{1}{g^{2}\left(x_{0}\right)}\left[f^{(2,0)}\left(x_{0}, y_{0}\right)+f^{(0,2)}\left(x_{0}, y_{0}\right)-f_{Y \mid x_{0}}\left(y_{0}\right) g^{\prime \prime}\left(x_{0}\right)\right]^{2} \\
& +\frac{A_{2,0}^{2}}{n h^{2}} \frac{f_{Y \mid x_{0}}\left(y_{0}\right)}{g\left(x_{0}\right)}+O\left(h^{6}\right)+O\left(\frac{1}{n h}\right) .
\end{aligned}
$$

The difference between the asymptotic variances of the naïve $\widehat{f}_{Y \mid x_{0}}$ and $\widehat{f}_{Y \mid x_{0}}^{\star}$ is whether the first term is divided by $g\left(x_{0}\right)$.

Remark 2 Under some regularity conditions, the $A M S E$ of $\widehat{f}_{Y \mid x_{0}}^{\dagger}\left(y_{0}\right)$ is given by

$$
\begin{aligned}
& E\left[\widehat{f}_{Y \mid x_{0}}^{\dagger}\left(y_{0}\right)-f_{Y \mid x_{0}}\left(y_{0}\right)\right]^{2} \\
= & \frac{h^{4} A_{1,2}^{2}}{4} \ell^{2}\left(y_{0}\right)\left[c^{(0,2)}\left(G\left(x_{0}\right), L\left(y_{0}\right)\right)+c^{(2,0)}\left(G\left(x_{0}\right), L\left(y_{0}\right)\right)\right]^{2} \\
& +\frac{A_{2,0}^{2}}{n h^{2}} f_{Y \mid x_{0}}\left(y_{0}\right) \ell\left(y_{0}\right)+O\left(h^{6}\right)+O\left(\frac{1}{n h}\right)
\end{aligned}
$$

where

$$
c^{(i, j)}\left(x_{0}, y_{0}\right)=\left.\left(\frac{\partial}{\partial w}\right)^{i}\left(\frac{\partial}{\partial z}\right)^{j} c(w, z)\right|_{w=x_{0}, z=y_{0}} .
$$


As the theorems and remarks show, the AMSEs of the conditional density function estimators are of the order $\left(h^{4}+\left(n h^{2}\right)^{-1}\right)$. Therefore, the theoretically optimal bandwidth is given by $n^{-1 / 6} \times C$ where $C>0$ is constant. For the following $A M S E$ :

$$
\frac{B}{4} h^{4}+\frac{V}{n h^{2}},
$$

the constant $C$ is given by $(2 V / B)^{-1 / 6}$. Determining the bandwidth needs to estimate the optimal one; however, it is quite difficult for conditional density estimators, as explained by Faugeras (2009). We postpone this for future work.

We present some results of a numerical study on conditional density estimation. We first compared the accuracy of $\widehat{f}_{Y \mid x_{0}}(y), \widehat{f}_{Y \mid x_{0}}^{\dagger}(y), \widehat{f}_{Y \mid x_{0}}^{\star}(y)$, and $\widehat{f}_{Y \mid x_{0}}^{\diamond}(y)$ at some fixed points $y=y_{0}$. Let us suppose that $(X, Y)$ is distributed normally, i.e., $f \sim N_{2}(\mathbf{0}, \Sigma)$. Set

$$
\Sigma=\left(\begin{array}{cc}
1 & \rho \sigma_{Y} \\
\rho \sigma_{Y} & \sigma_{Y}^{2}
\end{array}\right) .
$$

It follows that $g$ is also the normal density function, where the mean is 0 and variance is 1 . We simulated the mean squared error $(M S E)$, i.e.,

$$
\left(\widetilde{f}_{Y \mid x_{0}}\left(y_{0}\right)-f_{Y \mid x_{0}}\left(y_{0}\right)\right)^{2}
$$

and its standard deviation $(S D)$ for the conditional density estimator $\widetilde{f}_{Y \mid x_{0}}\left(y_{0}\right)$. Tables 1-3 show 100 times the obtained values. In the tables, 'Naïve', 'Product', 'Proposed', and 'Smoothed' denote $\widetilde{f}_{Y \mid x_{0}}\left(y_{0}\right)=\widehat{f}_{Y \mid x_{0}}\left(y_{0}\right), \widehat{f}_{Y \mid x_{0}}^{\dagger}\left(y_{0}\right), \widehat{f}_{Y \mid x_{0}}^{\star}\left(y_{0}\right)$, and $\widehat{f}_{Y \mid x_{0}}^{\diamond}\left(y_{0}\right)$ respectively. We simulated $M S E$ values (and their $S D$ ) of the conditional density estimators 100,000 times. The term $G^{-1}(\epsilon)$ denotes $x_{0}=G^{-1}(\epsilon)$, where $G^{-1}(\epsilon)$ is the $\epsilon$ th quantile of the distribution $G$ (of $X$, i.e., the standard normal distribution). The term $L^{-1}(\epsilon)$ denotes $y_{0}=L^{-1}(\epsilon)$, where $L^{-1}(\epsilon)$ is that of the distribution $L$ (of $Y$ ). In Tables 1-3, all the sample sizes are $(n=) 100$ and the bandwidths of the conditional density estimators are $n^{-1 / 6}$. From the results, we can first see that the smoothed version usually outperforms the proposed estimator. Table 1 suggests that the smoothed version of the proposed estimator gives almost best estimates especially when $\rho=0$ and $\sigma_{Y} \leq \sigma_{X}$. The naïve estimator is almost worst especially for $\sigma_{Y} \geq \sigma_{X}$. Table 2 also suggests that the naïve estimator is almost worst especially when $\rho=1 / 3$ and $\sigma_{Y} \geq \sigma_{X}$. As $\sigma_{Y}$ tends to be comparatively large, the product-shaped estimator becomes best. Table 3 suggests that the naïve one is most accurate especially when $\rho=2 / 3$ and $\sigma_{Y}<\sigma_{X}$. For $\sigma_{Y}>\sigma_{X}$, the product-shaped estimator is almost best. We summarized the obtained simulation results in Table 4 . The table shows a recommendation on nonparametric conditional density estimation for bivariate normal distributions. To sum up, the smoothed version of the proposed estimator seem to be superior to others especially when $\rho \approx 0$ and $\sigma_{Y} \leq \sigma_{X}$. When the correlation is high, the proposed estimators with $h=n^{-1 / 6}$ have large variance in some cases. Including the method of choosing bandwidth, we need to continue studying the proposed estimators. 
Table 1: MSE and SD values $(\times 100)$ with $\left(n=100, \rho=0, h=n^{-1 / 6}\right)$ for the conditional density estimators

\begin{tabular}{|c|c|c|c|c|c|c|c|c|c|}
\hline \multirow{2}{*}{$G^{-1}(\cdot)$} & \multirow{2}{*}{$L^{-1}(\cdot)$} & \multicolumn{2}{|c|}{ Naïve } & \multicolumn{2}{|c|}{ Product } & \multicolumn{2}{|c|}{ Proposed } & \multicolumn{2}{|c|}{ Smoothed } \\
\hline & & $M S E$ & $S D$ & $M S E$ & $S D$ & $M S E$ & $S D$ & $M S E$ & $S D$ \\
\hline \multicolumn{10}{|c|}{$\sigma_{Y}=1 / 2$} \\
\hline \multirow{3}{*}{$1 / 4$} & $1 / 4$ & 2.130 & 2.941 & 3.298 & 2.497 & 2.753 & 2.437 & 2.020 & 2.047 \\
\hline & $1 / 2$ & 2.249 & 3.128 & 3.150 & 2.363 & 3.208 & 2.658 & 2.356 & 2.232 \\
\hline & $3 / 4$ & 2.162 & 3.046 & 3.472 & 2.614 & 2.780 & 2.491 & 2.057 & 2.101 \\
\hline \multirow{3}{*}{$1 / 2$} & $1 / 4$ & 1.772 & 2.465 & 1.167 & 1.357 & 0.881 & 1.150 & 0.637 & 0.886 \\
\hline & $1 / 2$ & 1.823 & 2.522 & 0.919 & 1.125 & 0.976 & 1.263 & 0.646 & 0.909 \\
\hline & $3 / 4$ & 1.717 & 2.415 & 1.178 & 1.364 & 0.832 & 1.092 & 0.592 & 0.826 \\
\hline \multirow{3}{*}{$3 / 4$} & $1 / 4$ & 2.115 & 2.892 & 3.555 & 2.615 & 3.000 & 2.588 & 2.035 & 2.077 \\
\hline & $1 / 2$ & 2.226 & 3.066 & 3.468 & 2.592 & 3.536 & 2.891 & 2.393 & 2.311 \\
\hline & $3 / 4$ & 2.111 & 2.927 & 3.697 & 2.686 & 3.013 & 2.569 & 2.040 & 2.057 \\
\hline \multicolumn{10}{|c|}{$\sigma_{Y}=1$} \\
\hline \multirow{3}{*}{$1 / 4$} & $1 / 4$ & 326 & 1.895 & 0.920 & 0.817 & 0.600 & 0.742 & 0.514 & 0.668 \\
\hline & $1 / 2$ & 504 & 2.094 & 0.710 & 0.786 & 0.838 & 1.000 & 0.684 & 0.871 \\
\hline & $3 / 4$ & 1.308 & 1.867 & 0.975 & 0.862 & 0.597 & 0.746 & 0.510 & 0.665 \\
\hline \multirow{3}{*}{$1 / 2$} & $1 / 4$ & 1.062 & 1.542 & 0.510 & 0.599 & 0.403 & 0.584 & 0.460 & 0.700 \\
\hline & $1 / 2$ & 1.229 & 1.754 & 0.380 & 0.529 & 0.471 & 0.656 & 0.530 & 0.780 \\
\hline & $3 / 4$ & 1.050 & 1.473 & 0.535 & 0.619 & 0.405 & 0.573 & 0.460 & 0.679 \\
\hline \multirow{3}{*}{$3 / 4$} & $1 / 4$ & 1.306 & 1.911 & 0.958 & 0.834 & 0.636 & 0.771 & 0.515 & 0.669 \\
\hline & $1 / 2$ & 1.519 & 2.217 & 0.743 & 0.801 & 0.858 & 1.002 & 0.654 & 0.831 \\
\hline & $3 / 4$ & 1.315 & 1.885 & 1.011 & 0.866 & 0.626 & 0.752 & 0.507 & 0.652 \\
\hline \multicolumn{10}{|c|}{$\sigma_{Y}=2$} \\
\hline \multirow{3}{*}{$1 / 4$} & $1 / 4$ & 0.420 & 0.719 & 0.158 & 0.126 & 0.127 & 0.160 & 0.127 & 0.167 \\
\hline & $1 / 2$ & 901 & 1.337 & 0.253 & 0.312 & 0.337 & 0.423 & 0.308 & 0.401 \\
\hline & $3 / 4$ & 0.411 & 0.703 & 0.165 & 0.126 & 0.130 & 0.163 & 0.129 & 0.170 \\
\hline \multirow{3}{*}{$1 / 2$} & $1 / 4$ & 0.324 & 0.506 & 0.126 & 0.118 & 0.125 & 0.188 & 0.137 & 0.216 \\
\hline & $1 / 2$ & 0.742 & 1.115 & 0.223 & 0.330 & 0.280 & 0.405 & 0.315 & 0.484 \\
\hline & $3 / 4$ & 0.337 & 0.537 & 0.129 & 0.118 & 0.126 & 0.186 & 0.138 & 0.216 \\
\hline \multirow{3}{*}{$3 / 4$} & $1 / 4$ & 0.403 & 0.653 & 0.160 & 0.125 & 0.128 & 0.167 & 0.128 & 0.179 \\
\hline & $1 / 2$ & 935 & 1.368 & 0.260 & 0.311 & 0.350 & 0.435 & 0.314 & 0.409 \\
\hline & $3 / 4$ & 0.424 & 0.741 & 0.166 & 0.126 & 0.128 & 0.164 & 0.127 & 0.174 \\
\hline
\end{tabular}


Table 2: $\mathrm{MSE}$ and $\mathrm{SD}$ values $(\times 100)$ with $\left(n=100, \rho=1 / 3, h=n^{-1 / 6}\right)$ for the conditional density estimators

\begin{tabular}{|c|c|c|c|c|c|c|c|c|c|}
\hline \multirow{2}{*}{$G^{-1}(\cdot)$} & \multirow{2}{*}{$L^{-1}(\cdot)$} & \multicolumn{2}{|c|}{ Naïve } & \multicolumn{2}{|c|}{ Product } & \multicolumn{2}{|c|}{ Proposed } & \multicolumn{2}{|c|}{ Smoothed } \\
\hline & & $M S E$ & $S D$ & $M S E$ & $S D$ & $M S E$ & $S D$ & $M S E$ & $S D$ \\
\hline \multicolumn{10}{|c|}{$\sigma_{Y}=1 / 2$} \\
\hline \multirow{3}{*}{$1 / 4$} & $1 / 4$ & 2.427 & 3.289 & 4.788 & 3.050 & 7.691 & 4.547 & 6.242 & 4.061 \\
\hline & $1 / 2$ & 2.329 & 3.226 & 3.902 & 2.736 & 3.855 & 3.050 & 2.866 & 2.580 \\
\hline & $3 / 4$ & 1.991 & 2.753 & 3.369 & 2.518 & 0.964 & 1.260 & 0.698 & 0.970 \\
\hline \multirow{3}{*}{$1 / 2$} & $1 / 4$ & 1.826 & 2.522 & 1.680 & 1.726 & 1.144 & 1.406 & 0.735 & 1.013 \\
\hline & $1 / 2$ & 2.070 & 2.797 & 1.463 & 1.520 & 1.428 & 1.604 & 0.821 & 1.110 \\
\hline & $3 / 4$ & 1.881 & 2.610 & 1.753 & 1.773 & 1.221 & 1.518 & 0.750 & 1.075 \\
\hline \multirow{3}{*}{$3 / 4$} & $1 / 4$ & 2.021 & 2.853 & 3.458 & 2.585 & 1.077 & 1.361 & 0.717 & 0.976 \\
\hline & $1 / 2$ & 2.291 & 3.205 & 4.076 & 2.791 & 4.156 & 3.123 & 2.825 & 2.508 \\
\hline & $3 / 4$ & 2.318 & 3.171 & 5.135 & 3.200 & 8.157 & 4.639 & 6.225 & 4.030 \\
\hline \multicolumn{10}{|c|}{$\sigma_{Y}=1$} \\
\hline \multirow{3}{*}{$1 / 4$} & $1 / 4$ & 1.476 & 2.084 & 1.528 & 1.200 & 2.393 & 1.736 & 2.046 & 1.611 \\
\hline & $1 / 2$ & 546 & 2.181 & 0.831 & 0.878 & 0.922 & 1.072 & 0.741 & 0.924 \\
\hline & $3 / 4$ & 1.160 & 1.726 & 0.629 & 0.587 & 0.596 & 0.840 & 0.727 & 0.980 \\
\hline \multirow{3}{*}{$1 / 2$} & $1 / 4$ & 1.051 & 1.470 & 0.596 & 0.667 & 0.438 & 0.600 & 0.471 & 0.679 \\
\hline & $1 / 2$ & 1.251 & 1.702 & 0.447 & 0.615 & 0.520 & 0.713 & 0.517 & 0.748 \\
\hline & $3 / 4$ & 1.071 & 1.541 & 0.613 & 0.679 & 0.440 & 0.599 & 0.471 & 0.688 \\
\hline \multirow{3}{*}{$3 / 4$} & $1 / 4$ & 1.166 & 1.672 & 0.638 & 0.597 & 0.562 & 0.811 & 0.717 & 0.979 \\
\hline & $1 / 2$ & 1.548 & 2.152 & 0.883 & 0.914 & 1.006 & 1.134 & 0.752 & 0.932 \\
\hline & $3 / 4$ & 1.456 & 2.057 & 1.616 & 1.251 & 2.492 & 1.775 & 2.024 & 1.614 \\
\hline \multicolumn{10}{|c|}{$\sigma_{Y}=2$} \\
\hline \multirow{3}{*}{$1 / 4$} & $1 / 4$ & .528 & 0.824 & 0.304 & 0.213 & 0.381 & 0.285 & 0.350 & 0.278 \\
\hline & $1 / 2$ & 940 & 1.386 & 0.291 & 0.349 & 0.374 & 0.459 & 0.339 & 0.432 \\
\hline & $3 / 4$ & 0.282 & 0.519 & 0.051 & 0.050 & 0.282 & 0.412 & 0.307 & 0.439 \\
\hline \multirow{3}{*}{$1 / 2$} & $1 / 4$ & 0.302 & 0.501 & 0.105 & 0.105 & 0.129 & 0.198 & 0.143 & 0.228 \\
\hline & $1 / 2$ & 0.773 & 1.100 & 0.239 & 0.325 & 0.295 & 0.402 & 0.315 & 0.453 \\
\hline & $3 / 4$ & 0.314 & 0.517 & 0.108 & 0.105 & 0.131 & 0.194 & 0.147 & 0.229 \\
\hline \multirow{3}{*}{$3 / 4$} & $1 / 4$ & 0.287 & 0.538 & 0.050 & 0.051 & 0.283 & 0.408 & 0.314 & 0.439 \\
\hline & $1 / 2$ & 959 & 1.467 & 0.295 & 0.347 & 0.374 & 0.453 & 0.326 & 0.416 \\
\hline & $3 / 4$ & 0.525 & 0.866 & 0.316 & 0.213 & 0.393 & 0.292 & 0.352 & 0.281 \\
\hline
\end{tabular}


Table 3: MSE and SD values $(\times 100)$ with $\left(n=100, \rho=2 / 3, h=n^{-1 / 6}\right)$ for the conditional density estimators

\begin{tabular}{|c|c|c|c|c|c|c|c|c|c|}
\hline \multirow{2}{*}{$G^{-1}(\cdot)$} & \multirow{2}{*}{$L^{-1}(\cdot)$} & \multicolumn{2}{|c|}{ Naïve } & \multicolumn{2}{|c|}{ Product } & \multicolumn{2}{|c|}{ Proposed } & \multicolumn{2}{|c|}{ Smoothed } \\
\hline & & $M S E$ & $S D$ & $M S E$ & $S D$ & $M S E$ & $S D$ & $M S E$ & $S D$ \\
\hline \multicolumn{10}{|c|}{$\sigma_{Y}=1 / 2$} \\
\hline \multirow{3}{*}{$1 / 4$} & $1 / 4$ & 3.763 & 4.633 & 13.48 & 5.180 & 27.35 & 10.20 & 23.96 & 9.560 \\
\hline & $1 / 2$ & 2.441 & 3.427 & 6.344 & 3.687 & 6.019 & 4.371 & 4.539 & 3.718 \\
\hline & $3 / 4$ & 1.784 & 2.578 & 1.850 & 1.732 & 1.756 & 1.849 & 2.498 & 2.294 \\
\hline \multirow{3}{*}{$1 / 2$} & $1 / 4$ & 2.788 & 3.499 & 6.409 & 3.615 & 4.142 & 3.443 & 2.826 & 2.844 \\
\hline & $1 / 2$ & 3.684 & 4.047 & 7.510 & 3.850 & 6.401 & 3.735 & 4.454 & 3.227 \\
\hline & $3 / 4$ & 2.686 & 3.418 & 6.419 & 3.707 & 4.343 & 3.669 & 2.818 & 2.884 \\
\hline \multirow{3}{*}{$3 / 4$} & $1 / 4$ & 1.798 & 2.517 & 1.864 & 1.736 & 1.576 & 1.759 & 2.495 & 2.309 \\
\hline & & 2.415 & 3.299 & 6.424 & 3.656 & 6.557 & 4.623 & 4.538 & 3.742 \\
\hline & $/ 4$ & 3.756 & 4.530 & 13.92 & 5.287 & 28.61 & 10.46 & 23.96 & 9.582 \\
\hline \multicolumn{10}{|c|}{$\sigma_{Y}=1$} \\
\hline \multirow{3}{*}{$1 / 4$} & $1 / 4$ & 1.752 & 2.373 & 4.611 & 2.444 & 10.70 & 3.929 & 9.750 & 3.825 \\
\hline & $1 / 2$ & 1.610 & 2.277 & 1.310 & 1.174 & 1.343 & 1.461 & 1.054 & 1.254 \\
\hline & $3 / 4$ & 0.877 & 1.417 & 0.146 & 0.216 & 4.357 & 2.770 & 4.849 & 2.978 \\
\hline \multirow{3}{*}{$1 / 2$} & $1 / 4$ & 1.097 & 1.529 & 0.872 & 0.855 & 0.614 & 0.828 & 0.600 & 0.842 \\
\hline & $1 / 2$ & 1.484 & 2.073 & 1.569 & 1.466 & 1.267 & 1.406 & 0.953 & 1.184 \\
\hline & $3 / 4$ & 1.113 & 1.590 & 0.905 & 0.888 & 0.670 & 0.901 & 0.626 & 0.885 \\
\hline \multirow{3}{*}{$3 / 4$} & $1 / 4$ & 0.909 & 1.432 & 0.149 & 0.221 & 4.160 & 2.726 & 4.768 & 2.976 \\
\hline & $1 / 2$ & 1.610 & 2.250 & 1.341 & 1.180 & 1.447 & 1.488 & 1.029 & 1.199 \\
\hline & $3 / 4$ & 1.782 & 2.457 & 4.782 & 2.499 & 11.13 & 3.961 & 9.810 & 3.845 \\
\hline \multicolumn{10}{|c|}{$\sigma_{Y}=2$} \\
\hline \multirow{3}{*}{$1 / 4$} & $1 / 4$ & 0.641 & 1.004 & 0.501 & 0.330 & 1.129 & 0.397 & 1.070 & 0.404 \\
\hline & $1 / 2$ & 014 & 1.536 & 0.406 & 0.437 & 0.470 & 0.549 & 0.410 & 0.501 \\
\hline & $3 / 4$ & 0.088 & 0.240 & 0.025 & 0.038 & 1.050 & 0.914 & 1.091 & 0.943 \\
\hline \multirow{3}{*}{$1 / 2$} & $1 / 4$ & 0.230 & 0.445 & 0.045 & 0.068 & 0.172 & 0.308 & 0.193 & 0.336 \\
\hline & $1 / 2$ & 0.917 & 1.268 & 0.520 & 0.588 & 0.482 & 0.589 & 0.424 & 0.546 \\
\hline & $3 / 4$ & 0.233 & 0.429 & 0.043 & 0.064 & 0.163 & 0.296 & 0.195 & 0.340 \\
\hline \multirow{3}{*}{$3 / 4$} & $1 / 4$ & 0.092 & 0.271 & 0.024 & 0.037 & 1.030 & 0.899 & 1.065 & 0.923 \\
\hline & $1 / 2$ & .003 & 1.488 & 0.411 & 0.441 & 0.502 & 0.578 & 0.414 & 0.511 \\
\hline & $3 / 4$ & 0.638 & 0.994 & 0.510 & 0.326 & 1.145 & 0.390 & 1.063 & 0.403 \\
\hline
\end{tabular}


Table 4: Recommendation on nonparametric conditional density estimation for $\sigma_{X}=1$

\begin{tabular}{|c|c|c|c|c|}
\hline$G^{-1}(\cdot)$ & $L^{-1}(\cdot)$ & $\sigma_{Y}=1 / 2$ & $\sigma_{Y}=1$ & $\sigma_{Y}=2$ \\
\hline \multicolumn{5}{|c|}{$\rho=0$} \\
\hline \multirow{3}{*}{$1 / 4$} & $1 / 4$ & Smoothed & \multirow{3}{*}{ Smoothed } & Proposed \\
\hline & $1 / 2$ & Naïve & & Product \\
\hline & $3 / 4$ & Smoothed & & Smoothed \\
\hline \multirow{3}{*}{$1 / 2$} & $1 / 4$ & \multirow{3}{*}{ Smoothed } & Proposed & Proposed \\
\hline & $1 / 2$ & & Product & Product \\
\hline & $3 / 4$ & & Proposed & Proposed \\
\hline \multirow{3}{*}{$3 / 4$} & $1 / 4$ & Smoothed & \multirow{3}{*}{ Smoothed } & Proposed \\
\hline & $1 / 2$ & Naïve & & Product \\
\hline & $3 / 4$ & Smoothed & & Smoothed \\
\hline \multicolumn{5}{|c|}{$\rho=1 / 3$} \\
\hline \multirow{3}{*}{$1 / 4$} & $1 / 4$ & Naïve & Naïve & \multirow{3}{*}{ Product } \\
\hline & $1 / 2$ & Naïve & Smoothed & \\
\hline & $3 / 4$ & Smoothed & Proposed & \\
\hline \multirow{3}{*}{$1 / 2$} & $1 / 4$ & & Proposed & \multirow{3}{*}{ Product } \\
\hline & $1 / 2$ & Smoothed & Product & \\
\hline & $3 / 4$ & & Proposed & \\
\hline \multirow{3}{*}{$3 / 4$} & $1 / 4$ & Smoothed & Proposed & \multirow{3}{*}{ Product } \\
\hline & $1 / 2$ & Naïve & Smoothed & \\
\hline & $3 / 4$ & Naïve & Naïve & \\
\hline \multicolumn{5}{|c|}{$\rho=2 / 3$} \\
\hline \multirow{3}{*}{$1 / 4$} & $1 / 4$ & Naïve & Naïve & \multirow{3}{*}{ Product } \\
\hline & $1 / 2$ & Naïve & Smoothed & \\
\hline & $3 / 4$ & Proposed & Product & \\
\hline \multirow{3}{*}{$1 / 2$} & $1 / 4$ & \multirow{3}{*}{ Naïve } & \multirow{3}{*}{ Smoothed } & Product \\
\hline & $1 / 2$ & & & Smoothed \\
\hline & $3 / 4$ & & & Product \\
\hline \multirow{3}{*}{$3 / 4$} & $1 / 4$ & Proposed & Product & \multirow{3}{*}{ Product } \\
\hline & $1 / 2$ & Naïve & Smoothed & \\
\hline & $3 / 4$ & Naïve & Naïve & \\
\hline
\end{tabular}


Next, we consider to construct new regression function estimators. The regression function of $Y$ on $X=x_{0}$ is defined as the following integral:

$$
m\left(x_{0}\right):=E\left[Y \mid X=x_{0}\right]=\int y f_{Y \mid x_{0}}(y) \mathrm{d} y,
$$

that is, the expectation of $Y$ given $X=x_{0}$. It holds that

$$
\begin{aligned}
\int y \widehat{f}_{Y \mid x_{0}}(y) \mathrm{d} y & =\frac{1}{n h h \widehat{g}\left(x_{0}\right)} \int \sum_{i=1}^{n} y K\left(\frac{x_{0}-X_{i}}{h}\right) K\left(\frac{y-Y_{i}}{h}\right) \mathrm{d} y \\
& =\frac{1}{n h \widehat{g}\left(x_{0}\right)} \sum_{i=1}^{n} K\left(\frac{x_{0}-X_{i}}{h}\right) \int\left(Y_{i}+h u\right) K(u) \mathrm{d} u \\
& =\frac{1}{n h \widehat{g}\left(x_{0}\right)} \sum_{i=1}^{n} Y_{i} K\left(\frac{x_{0}-X_{i}}{h}\right) \\
& =: \widehat{m}\left(x_{0}\right),
\end{aligned}
$$

where $\widehat{m}$ is the Nadaraya-Watson estimator. Asymptotic properties of the NadarayaWatson estimator have been studied and summarized, for example, by

Wand and Jones (1995). Some asymptotic properties in multivariate settings were given by Georgiev (1988). Lei and Wasserman (2014) studied nonparametric prediction bands in a finite sample, and Burman and Chen (1989) introduced a nonparametric estimator for the heteroscedastic regression model. We fix the point $X=x_{0}$ throughout the paper; thus, we do not consider heteroscedasticity.

By the following integral of $\widehat{f}_{Y \mid x_{0}}^{\star}(y)$ and $\widehat{f}_{Y \mid x_{0}}^{\diamond}(y)$ :

$$
\begin{aligned}
\int y \widehat{f}_{Y \mid x_{0}}^{\star}(y) \mathrm{d} y & =\frac{1}{n h^{2}} \int \sum_{i=1}^{n} y K\left(\frac{Y_{i}-y}{h}\right) K\left(\frac{G_{n}\left(X_{i}\right)-G_{n}\left(x_{0}\right)}{h}\right) \mathrm{d} y \\
& =\frac{1}{n h} \sum_{i=1}^{n} \int\left(Y_{i}-h u\right) K(u) K\left(\frac{G_{n}\left(X_{i}\right)-G_{n}\left(x_{0}\right)}{h}\right) \mathrm{d} u \\
& =\frac{1}{n h} \sum_{i=1}^{n} Y_{i} K\left(\frac{G_{n}\left(X_{i}\right)-G_{n}\left(x_{0}\right)}{h}\right)
\end{aligned}
$$

we also propose the following regression function estimators:

$$
\widehat{m}^{\star}\left(x_{0}\right):=\frac{1}{n h} \sum_{i=1}^{n} Y_{i} K\left(\frac{G_{n}\left(X_{i}\right)-G_{n}\left(x_{0}\right)}{h}\right)
$$

and

$$
\widehat{m}^{\diamond}\left(x_{0}\right):=\frac{1}{n h} \sum_{i=1}^{n} Y_{i} K\left(\frac{\widehat{G}\left(X_{i}\right)-\widehat{G}\left(x_{0}\right)}{h}\right) .
$$

The next theorems show the AMSEs of these regression function estimators. 
Theorem 3 Let us assume the assumptions of Theorem 1 and that $V\left[Y \mid X=x_{0}\right]$ exists. Then, the AMSE of $\widehat{m}^{\star}\left(x_{0}\right)$ is given by

$$
\begin{aligned}
& E\left[\widehat{m}^{\star}\left(x_{0}\right)-m\left(x_{0}\right)\right]^{2} \\
= & \frac{h^{4} A_{1,2}^{2}}{4}\left[-m\left(x_{0}\right) \frac{g\left(x_{0}\right) g^{\prime \prime}\left(x_{0}\right)-3\left(g^{\prime}\left(x_{0}\right)\right)^{2}}{g^{4}\left(x_{0}\right)}\right. \\
& \left.\quad+\int z\left(\frac{3 g^{\prime}\left(x_{0}\right)}{g^{4}\left(x_{0}\right)} f^{(1,0)}\left(x_{0}, z\right)-\frac{1}{g^{3}\left(x_{0}\right)} f^{(2,0)}\left(x_{0}, z\right)\right) \mathrm{d} z\right]^{2} \\
& +\frac{A_{2,0}}{n h} V\left[Y \mid X=x_{0}\right]+O\left(h^{6}\right)+O\left(\frac{1}{n}\right) .
\end{aligned}
$$

Proof: $\quad$ The proof follows from Theorem 1.

Theorem 4 Under the assumptions of Theorem 3, the AMSE of $\widehat{m}^{\diamond}\left(x_{0}\right)$ is given by

$$
\begin{aligned}
& E\left[\widehat{m}^{\diamond}\left(x_{0}\right)-m\left(x_{0}\right)\right]^{2} \\
= & \frac{h^{4} A_{1,2}^{2}}{4}\left[m\left(x_{0}\right)\left(\frac{2 g\left(x_{0}\right) g^{\prime \prime}\left(x_{0}\right)}{g^{\prime}\left(x_{0}\right)}-\frac{g\left(x_{0}\right) g^{\prime \prime}\left(x_{0}\right)-3\left(g^{\prime}\left(x_{0}\right)\right)^{2}}{g^{4}\left(x_{0}\right)}\right)\right. \\
& \left.\quad+\int z\left(\frac{3 g^{\prime}\left(x_{0}\right)}{g^{4}\left(x_{0}\right)} f^{(1,0)}\left(x_{0}, z\right)-\frac{1}{g^{3}\left(x_{0}\right)} f^{(2,0)}\left(x_{0}, z\right)\right) \mathrm{d} z\right]^{2} \\
& +\frac{A_{2,0}}{n h} V\left[Y \mid X=x_{0}\right]+O\left(h^{6}\right)+O\left(\frac{1}{n}\right) .
\end{aligned}
$$

Proof: The proof follows from Theorem 2.

Remark 3 As seen above, the difference between the AMSEs of $\widehat{m}^{\star}\left(x_{0}\right)$ and $\widehat{m}^{\diamond}\left(x_{0}\right)$ is whether the first term in the bias terms exists. Under some regularity conditions, the $A M S E$ of the Nadaraya-Watson estimator $\widehat{m}\left(x_{0}\right)$ is given by

$$
\begin{aligned}
& E\left[\widehat{m}\left(x_{0}\right)-m\left(x_{0}\right)\right]^{2} \\
= & \frac{h^{4} A_{1,2}^{2}}{4} \frac{1}{g^{2}\left(x_{0}\right)}\left[-m\left(x_{0}\right) g^{\prime \prime}\left(x_{0}\right)+\int z f^{(2,0)}\left(x_{0}, z\right) \mathrm{d} z\right]^{2} \\
& +\frac{A_{2,0}}{n h} \frac{1}{g\left(x_{0}\right)} V\left[Y \mid X=x_{0}\right]+O\left(h^{6}\right)+O\left(\frac{1}{n}\right) .
\end{aligned}
$$

The difference between the asymptotic variances of $\widehat{m}\left(x_{0}\right)$ and $\widehat{m}^{\star}\left(x_{0}\right)$ is whether the first term is divided by $g\left(x_{0}\right)$. 
The AMSEs of the regression function estimators are of the order $\left(h^{4}+(n h)^{-1}\right)$, so the optimal bandwidth is of the order $n^{-1 / 5}$. For the following AMSE

$$
\frac{B}{4} h^{4}+\frac{V}{n h}
$$

the optimal bandwidth is given by $h=n^{-1 / 5} \times(V / B)^{-1 / 5}$.

Suppose that $F$ is the bivariate normal distribution $N_{2}\left(\mathbf{0}, \Sigma_{0}\right)$, where

$$
\Sigma_{0}=\left(\begin{array}{ll}
1 & \sigma \\
\sigma & 1
\end{array}\right)
$$

From the results, we can see that

$$
E\left[\widehat{m}^{\star}\left(x_{0}\right)-m\left(x_{0}\right)\right]^{2} \approx \frac{h^{4} A_{2,1}^{2}}{4} \frac{1}{\phi^{4}\left(x_{0}\right)}\left\{\frac{\rho x_{0}\left(2 \rho^{2}+1\right)}{1-\rho^{2}}\right\}^{2}+\frac{A_{0,2}}{n h}\left(1-\rho^{2}\right)
$$

and

$$
E\left[\widehat{m}\left(x_{0}\right)-m\left(x_{0}\right)\right]^{2} \approx \frac{h^{4} A_{2,1}^{2}}{4}\left(2 \rho x_{0}\right)^{2}+\frac{A_{0,2}}{n h} \frac{\left(1-\rho^{2}\right)}{\phi\left(x_{0}\right)},
$$

where $\phi\left(x_{0}\right)$ is the probability density of the standard normal distribution. Theoretically optimal bandwidths minimize $A M S E$ values, which equal

$$
\begin{gathered}
n^{-4 / 5} \frac{5}{4}\left[A_{2,1}\left(\frac{A_{0,2}}{4 \phi\left(x_{0}\right)}\right)^{2}\left(1-\rho^{2}\right)\left(\rho x_{0}\left(2 \rho^{2}+1\right)\right)\right]^{2 / 5} \\
n^{-4 / 5} \frac{5}{4}\left[A_{2,1}\left(\frac{A_{0,2}}{4 \phi\left(x_{0}\right)}\right)^{2}\left(1-\rho^{2}\right)^{2}\left(2 \rho x_{0}\right)\right]^{2 / 5} .
\end{gathered}
$$

It follows that the proposed estimator is theoretically better for $-\frac{1}{2}<\rho<\frac{1}{2}$ regardless of $x_{0}$ when $F \sim N_{2}(\mathbf{0}, \Sigma)$. The optimal bandwidths are

$$
\begin{gathered}
n^{-1 / 5}\left[\frac{A_{0,2}}{A_{2,1}^{2}} \phi^{4}\left(x_{0}\right)\left(1-\rho^{2}\right)\left\{\frac{1-\rho^{2}}{\rho x_{0}\left(2 \rho^{2}+1\right)}\right\}^{2}\right]^{1 / 5} \\
n^{-1 / 5}\left[\frac{A_{0,2}}{A_{2,1}^{2}} \frac{\left(1-\rho^{2}\right)\left(2 \rho x_{0}\right)^{2}}{\phi\left(x_{0}\right)}\right]^{1 / 5}
\end{gathered}
$$

respectively. We recommend choosing each bandwidth $h$ of the proposed estimators by a usual cross-validation procedure like the Nadaraya-Watson estimator.

We next compared the accuracy of $\widehat{m}\left(x_{0}\right), \widehat{m}^{\star}\left(x_{0}\right)$, and $\widehat{m}^{\diamond}\left(x_{0}\right)$. Suppose $f \sim$ $N_{2}(\mathbf{0}, \Sigma)$, where

$$
\Sigma=\left(\begin{array}{cc}
1 & \rho \sigma_{Y} \\
\rho \sigma_{Y} & \sigma_{Y}^{2}
\end{array}\right)
$$

We simulated the following mean squared error $(M S E)$ :

$$
\left(\widetilde{m}\left(x_{0}\right)-m\left(x_{0}\right)\right)^{2}
$$


and its standard deviation $(S D)$ for the regression estimator $\widetilde{m}\left(x_{0}\right)$. Tables 5 and 6 show 100 times the obtained values. The difference in the results between Tables 5 and 6 comes from the way to choose each bandwidth. In Table 5, the bandwidths of all regression estimators were fixed and $n^{-1 / 5}$. In Table 6 , they were data-driven and calculated by the cross-validation. $\widehat{h}$ denotes the cross-validation estimator. In the tables, 'Naïve', 'Proposed', and 'Smoothed' denote $\widetilde{m}\left(x_{0}\right)=\widehat{m}\left(x_{0}\right), \widehat{m}^{\star}\left(x_{0}\right)$, and $\widehat{m}^{\diamond}\left(x_{0}\right)$ respectively. All the sample sizes were $(n=) 100$, and we simulated $M S E$ values (and their $S D$ ) of the regression estimators 100,000 times. The cross-validated bandwidth in Table 6 was calculated at every time $(100,000)$ step. The term $G^{-1}(\epsilon)$ denotes $x_{0}=G^{-1}(\epsilon)$ defined before. We first see that the cross-validation is effective for estimating each optimal bandwidth by comparing Table 6 with Table 5. Compared with 'Smoothed', 'Proposed' is usually better. As seen from Table 6, 'Proposed' outperforms the others for $\rho=0$ regardless of both $x_{0}$ and $\sigma_{Y}$. Thus, the tables supports the above theoretical result that the proposed estimator theoretically outperforms the Nadaraya-Watson estimator for $\rho \approx 0$ regardless of $x_{0}$. Although the naïve Nadaraya-Watson estimator is better for $\rho=1 / 3$ and $2 / 3$, the difference is not so much. Therefore, we recommend 'Proposed' when the underlying probability distribution is bivariate normal and its correlation seems negligible. 
Table 5: MSE and SD values with $\left(n=100, h=n^{-1 / 5}\right)$ for the regression estimators

\begin{tabular}{|c|c|c|c|c|c|c|}
\hline \multirow{2}{*}{$G^{-1}(\cdot)$} & \multicolumn{2}{|c|}{ Naïve } & \multicolumn{2}{|c|}{ Proposed } & \multicolumn{2}{|c|}{ Smoothed } \\
\hline & $M S E$ & $S D$ & $M S E$ & $S D$ & $M S E$ & $S D$ \\
\hline \multicolumn{7}{|c|}{$\rho=0, \quad \sigma_{Y}=1 / 2$} \\
\hline $1 / 4$ & 0.178 & 0.196 & 0.137 & 0.109 & 0.138 & 0.112 \\
\hline $1 / 2$ & 0.062 & 0.091 & 0.024 & 0.033 & 0.025 & 0.036 \\
\hline $3 / 4$ & 0.180 & 0.197 & 0.138 & 0.109 & 0.139 & 0.112 \\
\hline \multicolumn{7}{|c|}{$\rho=0, \quad \sigma_{Y}=1$} \\
\hline $1 / 4$ & 0.767 & 0.880 & 0.545 & 0.426 & 0.548 & 0.433 \\
\hline $1 / 2$ & 0.248 & 0.357 & 0.095 & 0.134 & 0.101 & 0.143 \\
\hline $3 / 4$ & 0.765 & 0.878 & 0.546 & 0.424 & 0.550 & 0.434 \\
\hline \multicolumn{7}{|c|}{$\rho=0, \quad \sigma_{Y}=2$} \\
\hline $1 / 4$ & 4.316 & 5.701 & 2.088 & 1.447 & 2.088 & 1.446 \\
\hline $1 / 2$ & 0.990 & 1.433 & 0.378 & 0.537 & 0.403 & 0.572 \\
\hline $3 / 4$ & 4.363 & 5.787 & 2.081 & 1.434 & 2.089 & 1.456 \\
\hline \multicolumn{7}{|c|}{$\rho=1 / 3, \quad \sigma_{Y}=1 / 2$} \\
\hline $1 / 4$ & 0.056 & 0.080 & 0.031 & 0.044 & 0.032 & 0.046 \\
\hline $1 / 2$ & 0.097 & 0.128 & 0.271 & 0.171 & 0.266 & 0.172 \\
\hline $3 / 4$ & 0.228 & 0.233 & 0.879 & 0.282 & 0.872 & 0.287 \\
\hline \multicolumn{7}{|c|}{$\rho=1 / 3, \quad \sigma_{Y}=1$} \\
\hline $1 / 4$ & 0.241 & 0.350 & 0.531 & 0.464 & 0.545 & 0.475 \\
\hline $1 / 2$ & 0.239 & 0.347 & 0.540 & 0.466 & 0.527 & 0.467 \\
\hline $3 / 4$ & 0.407 & 0.593 & 2.540 & 0.868 & 2.544 & 0.885 \\
\hline \multicolumn{7}{|c|}{$\rho=1 / 3, \quad \sigma_{Y}=2$} \\
\hline $1 / 4$ & 1.893 & 2.698 & 4.874 & 2.409 & 4.890 & 2.433 \\
\hline $1 / 2$ & 0.987 & 1.423 & 1.403 & 1.447 & 1.368 & 1.449 \\
\hline $3 / 4$ & 4.966 & 7.697 & 7.691 & 2.566 & 7.735 & 2.592 \\
\hline \multicolumn{7}{|c|}{$\rho=2 / 3, \quad \sigma_{Y}=1 / 2$} \\
\hline $1 / 4$ & 0.041 & 0.059 & 0.462 & 0.272 & 0.446 & 0.269 \\
\hline $1 / 2$ & 0.060 & 0.083 & 1.404 & 0.373 & 1.386 & 0.380 \\
\hline $3 / 4$ & 0.095 & 0.126 & 2.470 & 0.411 & 2.470 & 0.423 \\
\hline \multicolumn{7}{|c|}{$\rho=2 / 3, \quad \sigma_{Y}=1$} \\
\hline $1 / 4$ & 0.140 & 0.202 & 0.216 & 0.301 & 0.215 & 0.299 \\
\hline $1 / 2$ & 0.349 & 0.434 & 2.929 & 1.066 & 2.877 & 1.086 \\
\hline $3 / 4$ & 1.046 & 1.127 & 6.030 & 1.153 & 6.077 & 1.178 \\
\hline \multicolumn{7}{|c|}{$\rho=2 / 3, \quad \sigma_{Y}=2$} \\
\hline $1 / 4$ & 3.044 & 2.775 & 7.670 & 3.451 & 7.711 & 3.497 \\
\hline $1 / 2$ & 2.939 & 2.721 & 7.672 & 3.422 & 7.511 & 3.481 \\
\hline $3 / 4$ & 24.99 & 21.55 & 16.47 & 3.354 & 16.47 & 3.369 \\
\hline
\end{tabular}


Table 6: MSE and SD values with $(n=100, h=\widehat{h})$ for the regression estimators

\begin{tabular}{|c|c|c|c|c|c|c|}
\hline \multirow{2}{*}{$G^{-1}(\cdot)$} & \multicolumn{2}{|c|}{ Naïve } & \multicolumn{2}{|c|}{ Proposed } & \multicolumn{2}{|c|}{ Smoothed } \\
\hline & $M S E$ & $S D$ & $M S E$ & $S D$ & $M S E$ & $S D$ \\
\hline \multicolumn{7}{|c|}{$\rho=0, \quad \sigma_{Y}=1 / 2$} \\
\hline $1 / 4$ & 0.115 & 0.022 & 0.114 & 0.020 & 0.117 & 0.040 \\
\hline $1 / 2$ & 0.001 & 0.003 & 0.001 & 0.002 & 0.004 & 0.007 \\
\hline $3 / 4$ & 0.115 & 0.022 & 0.115 & 0.020 & 0.117 & 0.040 \\
\hline \multicolumn{7}{|c|}{$\rho=0, \quad \sigma_{Y}=1$} \\
\hline $1 / 4$ & 0.459 & 0.088 & 0.458 & 0.078 & 0.468 & 0.160 \\
\hline $1 / 2$ & 0.004 & 0.014 & 0.003 & 0.010 & 0.014 & 0.029 \\
\hline $3 / 4$ & 0.459 & 0.090 & 0.458 & 0.078 & 0.469 & 0.160 \\
\hline \multicolumn{7}{|c|}{$\rho=0, \quad \sigma_{Y}=2$} \\
\hline $1 / 4$ & 1.838 & 0.380 & 1.832 & 0.319 & 1.864 & 0.598 \\
\hline $1 / 2$ & 0.017 & 0.058 & 0.013 & 0.039 & 0.056 & 0.117 \\
\hline $3 / 4$ & 1.839 & 0.377 & 1.833 & 0.314 & 1.868 & 0.599 \\
\hline \multicolumn{7}{|c|}{$\rho=1 / 3, \quad \sigma_{Y}=1 / 2$} \\
\hline $1 / 4$ & 0.001 & 0.004 & 0.001 & 0.002 & 0.004 & 0.007 \\
\hline $1 / 2$ & 0.108 & 0.020 & 0.114 & 0.020 & 0.125 & 0.041 \\
\hline $3 / 4$ & 0.433 & 0.044 & 0.457 & 0.039 & 0.486 & 0.076 \\
\hline \multicolumn{7}{|c|}{$\rho=1 / 3, \quad \sigma_{Y}=1$} \\
\hline $1 / 4$ & 0.111 & 0.038 & 0.123 & 0.043 & 0.152 & 0.090 \\
\hline $1 / 2$ & 0.106 & 0.037 & 0.118 & 0.042 & 0.146 & 0.090 \\
\hline $3 / 4$ & 0.952 & 0.136 & 1.044 & 0.117 & 1.146 & 0.232 \\
\hline \multicolumn{7}{|c|}{$\rho=1 / 3, \quad \sigma_{Y}=2$} \\
\hline $1 / 4$ & 0.916 & 0.244 & 1.097 & 0.253 & 1.322 & 0.518 \\
\hline $1 / 2$ & 0.110 & 0.072 & 0.132 & 0.096 & 0.209 & 0.223 \\
\hline $3 / 4$ & 2.552 & 0.469 & 2.954 & 0.402 & 3.319 & 0.800 \\
\hline \multicolumn{7}{|c|}{$\rho=2 / 3, \quad \sigma_{Y}=1 / 2$} \\
\hline $1 / 4$ & 0.101 & 0.019 & 0.112 & 0.021 & 0.126 & 0.037 \\
\hline $1 / 2$ & 0.419 & 0.048 & 0.455 & 0.039 & 0.486 & 0.067 \\
\hline $3 / 4$ & 0.962 & 0.077 & 1.027 & 0.057 & 1.076 & 0.098 \\
\hline \multicolumn{7}{|c|}{$\rho=2 / 3, \quad \sigma_{Y}=1$} \\
\hline $1 / 4$ & 0.003 & 0.010 & 0.004 & 0.011 & 0.014 & 0.031 \\
\hline $1 / 2$ & 0.397 & 0.087 & 0.468 & 0.082 & 0.534 & 0.143 \\
\hline $3 / 4$ & 1.664 & 0.200 & 1.856 & 0.156 & 1.993 & 0.275 \\
\hline \multicolumn{7}{|c|}{$\rho=2 / 3, \quad \sigma_{Y}=2$} \\
\hline $1 / 4$ & 0.382 & 0.145 & 0.519 & 0.180 & 0.675 & 0.329 \\
\hline $1 / 2$ & 0.365 & 0.139 & 0.497 & 0.179 & 0.648 & 0.321 \\
\hline $3 / 4$ & 3.588 & 0.576 & 4.254 & 0.484 & 4.691 & 0.876 \\
\hline
\end{tabular}




\section{Acknowledgement}

The authors thank the editor's and referees' valuable comments, which helped us improve this manuscript significantly. The authors gratefully acknowledge the Japan Society for the Promotion of Science, JSPS KAKENHI Grant Numbers JP16H02790, JP19K20223.

\section{References}

Bashtannyk, D. M. and Hyndman, R. J. (2001). Estimating and visualizing conditional densities, Computational Statistics 85 Data Analysis, 36(3), 279-298.

Burman, P. and Chen, K. W. (1989). Nonparametric estimation of a regression function, The Annals of Statistics, 1567-1596.

Ćwik, J. and Mielniczuk, J. (1989). Estimating density ratio with application to discriminant analysis, Communications in Statistics-Theory and Methods, 18(8), 30573069 .

Faugeras, O. P. (2009). A quantile-copula approach to conditional density estimation, Journal of Multivariate Analysis, 100(9), 2083-2099.

Georgiev, A. A. (1988). Asymptotic properties of the multivariate nadaraya-watson regression function estimate: the fixed design case, Statistics $\&$ probability letters, $7(1)$, $35-40$.

Hall, P., Wolff, R. C., and Yao, Q. (1999). Methods for estimating a conditional distribution function, Journal of the American Statistical Association, 94(445), 154-163.

Hall, P., Racine, J., and Li, Q. (2004). Cross-validation and the estimation of conditional probability densities, Journal of the American Statistical Association, 99(468), 1015-1026.

Hyndman, R. J., Bashtannyk, D. M., and Grunwald, G. K. (1996). Reaction and Control I. Mixing Additive and Multiplicative Network Algebras, Journal of Computational and Graphical Statistics, 5(4), 315-336.

Lei, J. and Wasserman, L. (2014). Distribution-free prediction bands for nonparametric regression, Journal of the Royal Statistical Society: Series B (Statistical Methodology), 76(1), 71-96.

Otneim, H. and Tjøstheim, D. (2017). Conditional density estimation using the local gaussian correlation, Statistics and Computing, 28(2), 301-321.

Rosenblatt, M. (1969). Conditional probability density and regression estimators, in Multivariate analysis II (P. R. Krishnaiah, ed.), 25-31. Academic Press, New York.

Ruppert, D. and Cline, D. B. H. (1994). Bias reduction in kernel density estimation by smoothed empirical transformations, The Annals of Statistics, 22(1), 185-210.

Vieu, P. (1991). Nonparametric regression: Optimal local bandwidth choice. Journal of the Royal Statistical Society Series B (Methodological), 53(2), 453-464.

Wand, M. P. and Jones M. C. (1995). Kernel Smoothing, Chapman and Hall, London. 


\section{Appendix: Proofs of Theorems}

\section{Proof of Theorem 1}

Suppose that the sample size $n$ is large enough, which ensures

$$
\min \left\{\frac{G\left(x_{0}\right)}{h}, \frac{1-G\left(x_{0}\right)}{h}\right\}>d
$$

The following expansion holds:

$$
\begin{aligned}
\widehat{f}_{Y \mid x_{0}}^{\star}\left(y_{0}\right)= & \frac{1}{n h^{2}} \sum_{i=1}^{n} K\left(\frac{Y_{i}-y_{0}}{h}\right) K\left(\frac{G\left(X_{i}\right)-G\left(x_{0}\right)}{h}\right) \\
& +\frac{1}{n h^{3}} \sum_{i=1}^{n} K\left(\frac{Y_{i}-y_{0}}{h}\right) K^{\prime}\left(\frac{G\left(X_{i}\right)-G\left(x_{0}\right)}{h}\right) \\
& +\frac{1}{n h^{4}} \sum_{i=1}^{n} K\left(\frac{Y_{i}-y_{0}}{h}\right) K^{\prime \prime}\left(\frac{G\left(X_{i}\right)-G\left(x_{0}\right)}{h}\right) \\
& +\frac{1}{n h^{5}} \sum_{i=1}^{n} K\left(\frac{Y_{i}-y_{0}}{h}\right) K^{(3)}\left(\frac{G_{n}{ }^{*}\left(X_{i}\right)-G_{n}{ }^{*}\left(x_{0}\right)}{h}\right) \\
& \left\{\left[G_{n}\left(X_{i}\right)-G\left(X_{i}\right)\right]-\left[G_{n}\left(x_{0}\right)-G\left(x_{0}\right)\right]\right\}^{3} \\
=: & \left.\left.\left.J_{1}+J_{2}+J_{3}+J_{4}{ }^{*}\right)\right]-\left[G_{n}\left(x_{0}\right)-G\left(x_{0}\right)\right]\right\}^{2}
\end{aligned}
$$

where $G_{n}{ }^{*}\left(X_{i}\right)$ is an r.v. between $G_{n}\left(X_{i}\right)$ and $G\left(X_{i}\right)$ with probability 1 and $G_{n}{ }^{*}\left(x_{0}\right)$ is between $G_{n}\left(x_{0}\right)$ and $G\left(x_{0}\right)$ with probability 1 .

First, we evaluate $J_{1}$, which is the sum of $i . i . d$.r.v.s. Let us use $\psi(u):=G^{-1}\left(G\left(x_{0}\right)+\right.$ $h u$ ). The expectation of $J_{1}$ is obtained as follows:

$$
\begin{aligned}
& E\left[J_{1}\right]=\frac{1}{h^{2}} \iint K\left(\frac{z-y_{0}}{h}\right) K\left(\frac{G(w)-G\left(x_{0}\right)}{h}\right) f(w, z) \mathrm{d} z \mathrm{~d} w \\
= & \frac{1}{h} \int K\left(\frac{z-y_{0}}{h}\right) \mathrm{d} z \int K(u) \frac{f(\psi(u), z)}{g(\psi(u))} \mathrm{d} u \\
= & \frac{1}{h} \int K\left(\frac{z-y_{0}}{h}\right)\left[\frac{f\left(x_{0}, z\right)}{g\left(x_{0}\right)}-\left\{f\left(x_{0}, z\right) \frac{g\left(x_{0}\right) g^{\prime \prime}\left(x_{0}\right)-3\left(g^{\prime}\left(x_{0}\right)\right)^{2}}{2 g^{5}\left(x_{0}\right)}-f^{(1,0)}\left(x_{0}, z\right) \frac{g^{\prime}\left(x_{0}\right)}{g^{4}\left(x_{0}\right)}\right.\right. \\
& \left.\left.+\frac{1}{2 g^{4}\left(x_{0}\right)}\left(g\left(x_{0}\right) f^{(2,0)}\left(x_{0}, z\right)-g^{\prime}\left(x_{0}\right) f^{(1,0)}\left(x_{0}, z\right)\right)\right\} h^{2} A_{1,2}\right] \mathrm{d} z+O\left(h^{4}\right) \\
= & \int K(v)\left[\left\{\frac{1}{g\left(x_{0}\right)} f\left(x_{0}, y_{0}\right)+f^{(0,2)}\left(x_{0}, y_{0}\right) \frac{(h v)^{2}}{2}\right\}-\left\{f\left(x_{0}, y_{0}\right) \frac{g\left(x_{0}\right) g^{\prime \prime}\left(x_{0}\right)-3\left(g^{\prime}\left(x_{0}\right)\right)^{2}}{2 g^{5}\left(x_{0}\right)}\right.\right. \\
& \left.\left.-f^{(1,0)}\left(x_{0}, y_{0}\right) \frac{g^{\prime}\left(x_{0}\right)}{g^{4}\left(x_{0}\right)}+\frac{1}{2 g^{4}\left(x_{0}\right)}\left(g\left(x_{0}\right) f^{(2,0)}\left(x_{0}, y_{0}\right)-g^{\prime}\left(x_{0}\right) f^{(1,0)}\left(x_{0}, y_{0}\right)\right)\right\} h^{2} A_{1,2}\right] \mathrm{d} v \\
& +O\left(h^{4}\right) .
\end{aligned}
$$


From the calculation of the following second moment:

$$
\begin{aligned}
& \frac{1}{n h^{4}} \iint K^{2}\left(\frac{z-y_{0}}{h}\right) K^{2}\left(\frac{G(w)-G\left(x_{0}\right)}{h}\right) f(w, z) \mathrm{d} z \mathrm{~d} w \\
= & \frac{1}{n h^{3}} \int K^{2}\left(\frac{z-y_{0}}{h}\right) K^{2}(u) \frac{f(\psi(u), z)}{g(\psi(u))} \mathrm{d} z \mathrm{~d} u \\
= & \frac{A_{2,0}^{2}}{n h^{2}} f_{Y \mid x_{0}}\left(y_{0}\right)+O\left(\frac{1}{n h}\right)
\end{aligned}
$$

we can see that

$$
V\left[J_{1}\right]=E\left[\left(J_{1}\right)^{2}\right]-E\left[J_{1}\right]^{2}=\frac{A_{2,0}^{2}}{n h^{2}} f_{Y \mid x_{0}}\left(y_{0}\right)+O\left(\frac{1}{n h}\right) .
$$

By applying a conditional expectation to $J_{2}$, we can obtain

$$
\begin{aligned}
& E\left[J_{2}\right]=\frac{1}{n^{2} h^{3}} \sum_{i=1}^{n} \sum_{j=1}^{n} E\left[K\left(\frac{Y_{i}-y_{0}}{h}\right) K^{\prime}\left(\frac{G\left(X_{i}\right)-G\left(x_{0}\right)}{h}\right)\right. \\
& \left.\left\{\left[I\left(X_{j} \leq X_{i}\right)-G\left(X_{i}\right)\right]-\left[I\left(X_{j} \leq x_{0}\right)-G\left(x_{0}\right)\right]\right\}\right] \\
= & \frac{1}{n h^{3}} E\left[K\left(\frac{Y_{i}-y_{0}}{h}\right) K^{\prime}\left(\frac{G\left(X_{i}\right)-G\left(x_{0}\right)}{h}\right)\right. \\
& \left.\quad \sum_{j=1}^{n} E\left[\left[I\left(X_{j} \leq X_{i}\right)-G\left(X_{i}\right)\right]-\left[I\left(X_{j} \leq x_{0}\right)-G\left(x_{0}\right)\right] \mid Y_{i}, X_{i}\right]\right] \\
\approx & \frac{1}{n h^{3}} E\left[K\left(\frac{Y_{i}-y_{0}}{h}\right) K^{\prime}\left(\frac{G\left(X_{i}\right)-G\left(x_{0}\right)}{h}\right)\left\{\left[1-G\left(X_{i}\right)\right]-\left[I\left(X_{i} \leq x_{0}\right)-G\left(x_{0}\right)\right]\right\}\right] \\
= & \frac{1}{n h^{2}} \iint K\left(\frac{z-y_{0}}{h}\right) K^{\prime}(u)\left\{[1-G(\psi(u))]-\left[I\left(\psi(u) \leq x_{0}\right)-G\left(x_{0}\right)\right]\right\} \frac{f(\psi(u), z)}{g(\psi(u))} \mathrm{d} z \mathrm{~d} u \\
= & O\left(\frac{1}{n}\right) .
\end{aligned}
$$

The squared value of $J_{2}$ is given by

$$
\begin{aligned}
&\left(J_{2}\right)^{2}= \frac{1}{n^{4} h^{6}} \sum_{i=1}^{n} \sum_{j=1}^{n} \sum_{k=1}^{n} \sum_{m=1}^{n} K\left(\frac{Y_{i}-y_{0}}{h}\right) K\left(\frac{Y_{k}-y_{0}}{h}\right) K^{\prime}\left(\frac{G\left(X_{i}\right)-G\left(x_{0}\right)}{h}\right) \\
& K^{\prime}\left(\frac{G\left(X_{k}\right)-G\left(x_{0}\right)}{h}\right)\left\{\left[I\left(X_{j} \leq X_{i}\right)-G\left(X_{i}\right)\right]-\left[I\left(X_{j} \leq x_{0}\right)-G\left(x_{0}\right)\right]\right\} \\
& \quad\left\{\left[I\left(X_{m} \leq X_{k}\right)-G\left(X_{k}\right)\right]-\left[I\left(X_{m} \leq x_{0}\right)-G\left(x_{0}\right)\right]\right\} \\
&=: \frac{1}{n^{4} h^{6}} \sum_{i=1}^{n} \sum_{j=1}^{n} \sum_{k=1}^{n} \sum_{m=1}^{n} \Xi(i, j, k, m) .
\end{aligned}
$$


We can find the following:

if all of $(i, j, k, m)$ are different, $E[\Xi(i, j, k, m)]=E\left[E\left[\Xi(i, j, k, m) \mid X_{i}, X_{k}\right]\right]=0$ if $i=j$ and all of $(i, k, m)$ are different, $E[\Xi(i, j, k, m)]=E\left[E\left[\Xi(i, j, k, m) \mid X_{i}, X_{k}\right]\right]=0$ if $i=k$ and all of $(i, j, m)$ are different, $E[\Xi(i, j, k, m)]=E\left[E\left[\Xi(i, j, k, m) \mid X_{i}\right]\right]=0$ if $i=m$ and all of $(i, j, k)$ are different, $E[\Xi(i, j, k, m)]=E\left[E\left[\Xi(i, j, k, m) \mid X_{i}\right]\right]=0$.

From the above results, we can see that the terms in which $j=m$ and all $(i, j, k)$ are different is the main of $E\left[\left(J_{2}\right)^{2}\right]$. For the term in which $j=m$ and all $(i, j, k)$ are different, the expectation is given by

$$
\begin{aligned}
& E[\Xi(i, j, k, m)] \\
= & \frac{n(n-1)(n-2)}{n^{4} h^{4}} E\left[K\left(\frac{Y_{i}-y_{0}}{h}\right) K\left(\frac{Y_{k}-y_{0}}{h}\right) K^{\prime}\left(\frac{G\left(X_{i}\right)-G\left(x_{0}\right)}{h}\right)\right. \\
& K^{\prime}\left(\frac{G\left(X_{k}\right)-G\left(x_{0}\right)}{h}\right)\left\{\left[I\left(X_{j} \leq X_{i}\right)-G\left(X_{i}\right)\right]-\left[I\left(X_{j} \leq x_{0}\right)-G\left(x_{0}\right)\right]\right\} \\
& \left.\left\{\left[I\left(X_{j} \leq X_{k}\right)-G\left(X_{k}\right)\right]-\left[I\left(X_{j} \leq x_{0}\right)-G\left(x_{0}\right)\right]\right\}\right] .
\end{aligned}
$$

It holds that

$$
\begin{aligned}
& E\left[E \left[\left\{\left[I\left(X_{j} \leq X_{i}\right)-G\left(X_{i}\right)\right]-\left[I\left(X_{j} \leq x_{0}\right)-G\left(x_{0}\right)\right]\right\}\right.\right. \\
& \left.\left.\quad \times\left\{\left[I\left(X_{j} \leq X_{k}\right)-G\left(X_{k}\right)\right]-\left[I\left(X_{j} \leq x_{0}\right)-G\left(x_{0}\right)\right]\right\} \mid X_{i}, X_{k}\right]\right] \\
& =E\left[G\left(\min \left(X_{i}, X_{k}\right)\right)-G\left(\min \left(X_{i}, x_{0}\right)\right)-G\left(\min \left(X_{k}, x_{0}\right)\right)-G\left(X_{i}\right) G\left(X_{k}\right)+G\left(X_{i}\right) G\left(x_{0}\right)\right. \\
& \left.\quad+G\left(X_{k}\right) G\left(x_{0}\right)-G^{2}\left(x_{0}\right)+G\left(x_{0}\right)\right] .
\end{aligned}
$$

Let us evaluate the following first term (with respect to $G\left(\min \left(X_{i}, X_{k}\right)\right)$ )

$$
\begin{aligned}
E[K & \left(\frac{Y_{i}-y_{0}}{h}\right) K\left(\frac{Y_{k}-y_{0}}{h}\right) K^{\prime}\left(\frac{G\left(X_{i}\right)-G\left(x_{0}\right)}{h}\right) K^{\prime}\left(\frac{G\left(X_{k}\right)-G\left(x_{0}\right)}{h}\right) \\
& \left.\times G\left(\min \left(X_{i}, X_{k}\right)\right)\right] \\
= & \cdots \int_{-\infty}^{v} K\left(\frac{z_{i}-y_{0}}{h}\right) K\left(\frac{z_{k}-y_{0}}{h}\right) K^{\prime}\left(\frac{G(w)-G\left(x_{0}\right)}{h}\right) K^{\prime}\left(\frac{G(v)-G\left(x_{0}\right)}{h}\right) \\
& \times G(w) f\left(w, z_{i}\right) f\left(v, z_{k}\right) \mathrm{d} w \mathrm{~d} v \mathrm{~d} z_{i} \mathrm{~d} z_{k} \\
+ & \cdots \int_{v}^{\infty} K\left(\frac{z_{i}-y_{0}}{h}\right) K\left(\frac{z_{k}-y_{0}}{h}\right) K^{\prime}\left(\frac{G(w)-G\left(x_{0}\right)}{h}\right) K^{\prime}\left(\frac{G(v)-G\left(x_{0}\right)}{h}\right) \\
& \times G(v) f\left(w, z_{i}\right) f\left(v, z_{k}\right) \mathrm{d} w \mathrm{~d} v \mathrm{~d} z_{i} \mathrm{~d} z_{k}
\end{aligned}
$$




$$
\begin{aligned}
=-h^{2} & \int \ldots \int K\left(\frac{z_{i}-y_{0}}{h}\right) K\left(\frac{z_{k}-y_{0}}{h}\right) W\left(\frac{G(v)-G\left(x_{0}\right)}{h}\right) K^{\prime}\left(\frac{G(v)-G\left(x_{0}\right)}{h}\right) \\
& \times\left(\frac{f\left(x_{0}, z_{i}\right)}{g\left(x_{0}\right)}+\frac{G\left(x_{0}\right)}{g^{3}\left(x_{0}\right)}\left(g\left(x_{0}\right) f^{(1,0)}\left(x_{0}, z_{i}\right)-f\left(x_{0}, z_{i}\right) g^{\prime}\left(x_{0}\right)\right)\right) f\left(v, z_{k}\right) \mathrm{d} v \mathrm{~d} z_{i} \mathrm{~d} z_{k} \\
- & h^{2} \int \cdots \int K\left(\frac{z_{i}-y_{0}}{h}\right) z_{k}\left(1-W\left(\frac{G(v)-G\left(x_{0}\right)}{h}\right)\right) K^{\prime}\left(\frac{G(v)-G\left(x_{0}\right)}{h}\right) \\
& \times \frac{G(v)}{g^{3}\left(x_{0}\right)}\left(g\left(x_{0}\right) f^{(1,0)}\left(x_{0}, z_{i}\right)-f\left(x_{0}, z_{i}\right) g^{\prime}\left(x_{0}\right)\right) f\left(v, z_{k}\right) \mathrm{d} v \mathrm{~d} z_{i} \mathrm{~d} z_{k}+O\left(h^{3}\right),
\end{aligned}
$$

where $W$ is the c.d.f. of $K$. It follows that

$$
\begin{aligned}
& E\left[K\left(\frac{Y_{i}-y_{0}}{h}\right) K\left(\frac{Y_{k}-y_{0}}{h}\right) K^{\prime}\left(\frac{G\left(X_{i}\right)-G\left(x_{0}\right)}{h}\right) K^{\prime}\left(\frac{G\left(X_{k}\right)-G\left(x_{0}\right)}{h}\right)\right. \\
& \left.\times G\left(\min \left(X_{i}, X_{k}\right)\right)\right] \\
& =h^{3} \iint K\left(\frac{z_{i}-y_{0}}{h}\right) K\left(\frac{z_{k}-y_{0}}{h}\right)\left(A_{2,0} \frac{f\left(x_{0}, z_{k}\right)}{g\left(x_{0}\right)}\right. \\
& \left.+\frac{h}{2 g^{3}\left(x_{0}\right)}\left(g\left(x_{0}\right) f^{(1,0)}\left(x_{0}, z_{k}\right)-f\left(x_{0}, z_{k}\right) g^{\prime}\left(x_{0}\right)\right)\right) \\
& \times\left(\frac{f\left(x_{0}, z_{i}\right)}{g\left(x_{0}\right)}+\frac{G\left(x_{0}\right)}{g^{3}\left(x_{0}\right)}\left(g\left(x_{0}\right) f^{(1,0)}\left(x_{0}, z_{i}\right)-f\left(x_{0}, z_{i}\right) g^{\prime}\left(x_{0}\right)\right)\right) \mathrm{d} z_{i} \mathrm{~d} z_{k} \\
& +h^{3} \iint z_{i} z_{k}\left(-A_{2,0} G\left(x_{0}\right) \frac{f\left(x_{0}, z_{k}\right)}{g\left(x_{0}\right)}\right. \\
& \left.+\frac{h}{2 g^{3}\left(x_{0}\right)} G\left(x_{0}\right)\left(g\left(x_{0}\right) f^{(1,0)}\left(x_{0}, z_{k}\right)-f\left(x_{0}, z_{k}\right) g^{\prime}\left(x_{0}\right)\right)+h \frac{f\left(x_{0}, z_{k}\right)}{2 g\left(x_{0}\right)}\right) \\
& \times \frac{1}{g^{3}\left(x_{0}\right)}\left(g\left(x_{0}\right) f^{(1,0)}\left(x_{0}, z_{i}\right)-f\left(x_{0}, z_{i}\right) g^{\prime}\left(x_{0}\right)\right) \mathrm{d} z_{i} \mathrm{~d} z_{k}+O\left(h^{4}\right) \\
& =h^{3} A_{2,0} \iint K\left(\frac{z_{i}-y_{0}}{h}\right) K\left(\frac{z_{k}-y_{0}}{h}\right) \frac{f\left(x_{0}, z_{k}\right)}{g\left(x_{0}\right)} \frac{f\left(x_{0}, z_{i}\right)}{g\left(x_{0}\right)} \mathrm{d} z_{i} \mathrm{~d} z_{k}+O\left(h^{4}\right) .
\end{aligned}
$$

By a similar calculation, we can see

$$
\begin{gathered}
E\left[K\left(\frac{Y_{i}-y_{0}}{h}\right) K\left(\frac{Y_{k}-y_{0}}{h}\right) K^{\prime}\left(\frac{G\left(X_{i}\right)-G\left(x_{0}\right)}{h}\right) K^{\prime}\left(\frac{G\left(X_{k}\right)-G\left(x_{0}\right)}{h}\right)\right. \\
\left.G\left(\min \left(X_{i}, x_{0}\right)\right)\right]=O\left(h^{4}\right)
\end{gathered}
$$

and

$$
\begin{gathered}
E\left[K\left(\frac{Y_{i}-y_{0}}{h}\right) K\left(\frac{Y_{k}-y_{0}}{h}\right) K^{\prime}\left(\frac{G\left(X_{i}\right)-G\left(x_{0}\right)}{h}\right) K^{\prime}\left(\frac{G\left(X_{k}\right)-G\left(x_{0}\right)}{h}\right)\right. \\
\left.G\left(X_{i}\right) G\left(X_{k}\right)\right]=O\left(h^{4}\right) .
\end{gathered}
$$

Therefore, it holds that for the term in which $j=m$ and all $(i, j, k)$ are different,

$$
E[\Xi(i, j, k, m)]=\frac{A_{2,0}}{n h} E[Y \mid x]^{2}
$$


and that the other combinations of $(i, j, k, m)$ are of the order $n^{-1}$. As a result, we obtain $E\left[\left(J_{2}\right)^{2}\right]=O\left((n h)^{-1}\right)$ and

$$
V\left[J_{2}\right]=\frac{A_{2,0}}{n h} E[Y \mid x]^{2} .
$$

The moments of $J_{3}$ are obtained in a similar manner, which are given by

$$
E\left[J_{3}\right]=O\left(n^{-1}\right) \text { and } V\left[J_{3}\right]=O\left(n^{-2} h^{-1}\right) .
$$

Finally, we obtain upper bounds of $\left|E\left[J_{4}^{*}\right]\right|$ and $E\left[\left(J_{4}^{*}\right)^{2}\right]$. From the assumption of Theorem 1, we can see that

$$
\begin{aligned}
E\left[J_{4}^{*}\right]= & \mid E\left[\frac{1}{h^{5}} K\left(\frac{Y_{i}-y_{0}}{h}\right) K^{(3)}\left(\frac{G_{n}^{*}\left(X_{i}\right)-G_{n}^{*}\left(x_{0}\right)}{h}\right)\right. \\
\left.\left\{\left[G_{n}\left(X_{i}\right)-G\left(X_{i}\right)\right]-\left[G_{n}\left(x_{0}\right)-G\left(x_{0}\right)\right]\right\}^{3}\right] \mid & \\
\leq & \frac{\max _{u} K(u) \times \max _{v}\left|K^{(3)}(v)\right|}{h^{5}} E\left[\left\{\left[G_{n}\left(X_{i}\right)-G\left(X_{i}\right)\right]-\left[G_{n}\left(x_{0}\right)-G\left(x_{0}\right)\right]\right\}^{3}\right] \\
= & O\left(\frac{1}{n^{2} h^{5}}\right) .
\end{aligned}
$$

Similarly, it follows that

$$
\begin{aligned}
& E\left[\left(J_{4}^{*}\right)^{2}\right] \\
\leq & \left(\frac{\max _{u} K(u) \times \max _{v}\left|K^{(3)}(v)\right|}{h^{5}}\right)^{2} E\left[\left\{\left[G_{n}\left(X_{i}\right)-G\left(X_{i}\right)\right]-\left[G_{n}\left(x_{0}\right)-G\left(x_{0}\right)\right]\right\}^{3}\right. \\
& \left.\quad \times\left\{\left[G_{n}\left(X_{j}\right)-G\left(X_{j}\right)\right]-\left[G_{n}\left(x_{0}\right)-G\left(x_{0}\right)\right]\right\}^{3}\right] \\
= & O\left(\frac{1}{n^{4} h^{10}}\right) .
\end{aligned}
$$

To sum up, we conclude that $J_{2}+J_{3}+J_{4}^{*}$ is asymptotically negligible for fixed $x_{0}$. The main bias of $\widehat{f}_{Y \mid x_{0}}^{\star}\left(y_{0}\right)$ comes from $J_{1}$. Since

$$
\begin{aligned}
E\left[J_{1} J_{2}\right]= & \frac{1}{n^{3} h^{3}} \sum_{i=1}^{n} \sum_{j=1}^{n} \sum_{k=1}^{n} K\left(\frac{Y_{i}-y}{h}\right) K\left(\frac{Y_{j}-y_{0}}{h}\right) K^{\prime}\left(\frac{G\left(X_{i}\right)-G\left(x_{0}\right)}{h}\right) \\
& K^{\prime}\left(\frac{G\left(X_{j}\right)-G\left(x_{0}\right)}{h}\right) \times\left\{\left[I\left(X_{k} \leq X_{j}\right)-G\left(X_{j}\right)\right]-\left[I\left(X_{k} \leq x_{0}\right)-G\left(x_{0}\right)\right]\right\} \\
= & E\left[J_{1}\right] E\left[J_{2}\right]+O\left(\frac{1}{n}\right),
\end{aligned}
$$

using the Cauchy-Schwarz inequality, the asymptotic variance is given by $V\left[J_{1}\right]$. Thus, we have

$$
V\left[\widehat{f}_{Y \mid x_{0}}^{\star}\left(y_{0}\right)\right]=\frac{A_{2,0}^{2}}{n h^{2}} f_{Y \mid x_{0}}\left(y_{0}\right)+O\left((n h)^{-1}\right)
$$


and Theorem 1 has been proved.

\section{Proof of Theorem 2}

The same as the proof of Theorem 1, the $A M S E$ of $\widehat{f}_{Y \mid x_{0}}^{\diamond}\left(y_{0}\right)$ is obtained. In a similar manner, we decompose $\widehat{f}_{Y \mid x_{0}}^{\diamond}\left(y_{0}\right)$ as follows:

$$
\begin{aligned}
\widehat{f}_{Y \mid x_{0}}^{\diamond}\left(y_{0}\right)= & \frac{1}{n h^{2}} \sum_{i=1}^{n} K\left(\frac{Y_{i}-y_{0}}{h}\right) K\left(\frac{\widehat{G}\left(X_{i}\right)-\widehat{G}\left(x_{0}\right)}{h}\right) \\
= & \frac{1}{n h^{2}} \sum_{i=1}^{n} K\left(\frac{Y_{i}-y_{0}}{h}\right) K\left(\frac{G\left(X_{i}\right)-G\left(x_{0}\right)}{h}\right) \\
& +\frac{1}{n h^{3}} \sum_{i=1}^{n} K\left(\frac{Y_{i}-y_{0}}{h}\right) K^{\prime}\left(\frac{G\left(X_{i}\right)-G\left(x_{0}\right)}{h}\right) \\
& +\frac{1}{n h^{4}} \sum_{i=1}^{n} K\left(\frac{Y_{i}-y_{0}}{h}\right) K^{\prime \prime}\left(\frac{G\left(X_{i}\right)-G\left(x_{0}\right)}{h}\right) \\
\left\{\left[\widehat{G}\left(X_{i}\right)-G\left(X_{i}\right)\right]-\left[\widehat{G}\left(x_{0}\right)-G\left(x_{0}\right)\right]\right\}^{2} & \frac{1}{n h^{5}} \sum_{i=1}^{n} K\left(\frac{Y_{i}-y_{0}}{h}\right) K^{(3)}\left(\frac{\widehat{G}^{*}\left(X_{i}\right)-G\left(x_{0}\right)}{h}\right) \\
\left\{\left[\widehat{G}\left(X_{i}\right)-G\left(X_{i}\right)\right]-\left[\widehat{G}\left(x_{0}\right)-G\left(x_{0}\right)\right]\right\}^{3} & \left.J_{1}+J_{2}^{\diamond}+J_{3}^{\diamond}+J_{4}^{\diamond *}\right)
\end{aligned}
$$

where $\widehat{G}^{*}\left(X_{i}\right)$ is an r.v. between $\widehat{G}\left(X_{i}\right)$ and $G\left(X_{i}\right)$ with probability 1 , and $G_{n}{ }^{*}\left(x_{0}\right)$ is between $G_{n}\left(x_{0}\right)$ and $G\left(x_{0}\right)$ with probability 1 . The moments of $J_{1}$ are given in the proof of Theorem 1 . We need to calculate the expectation of $J_{2}^{\diamond}$ again in this case. By using conditional expectation, we have the following:

$$
\begin{aligned}
& E\left[J_{2}^{\diamond}\right] \\
= & \frac{1}{n^{2} h^{3}} \sum_{i=1}^{n} \sum_{j=1}^{n} E\left[K\left(\frac{Y_{i}-y_{0}}{h}\right) K^{\prime}\left(\frac{G\left(X_{i}\right)-G\left(x_{0}\right)}{h}\right)\right. \\
& \left.\quad\left\{\left[W\left(\frac{X_{i}-X_{j}}{h}\right)-G\left(X_{i}\right)\right]-\left[W\left(\frac{x_{0}-X_{j}}{h}\right)-G\left(x_{0}\right)\right]\right\}\right] \\
= & \frac{1}{n h^{3}} E\left[K\left(\frac{Y_{i}-y_{0}}{h}\right) K^{\prime}\left(\frac{G\left(X_{i}\right)-G\left(x_{0}\right)}{h}\right)\right. \\
& \left.\times \sum_{j=1}^{n} E\left[\left\{W\left(\frac{X_{i}-X_{j}}{h}\right)-G\left(X_{i}\right)\right\}-\left\{W\left(\frac{x_{0}-X_{j}}{h}\right)-G\left(x_{0}\right)\right\} \mid Y_{i}, X_{i}\right]\right] \\
= & \frac{1}{n h^{3}} E\left[K\left(\frac{Y_{i}-y_{0}}{h}\right) K^{\prime}\left(\frac{G\left(X_{i}\right)-G\left(x_{0}\right)}{h}\right)\left[\left\{n h^{2} A_{1,2}\left[g^{\prime}\left(X_{i}\right)-g^{\prime}\left(x_{0}\right)\right]\right\}+O\left(n h^{4}+1\right)\right]\right] \\
= & \iint A_{1,2} K\left(\frac{z-y_{0}}{h}\right) K^{\prime}(u)\left[g^{\prime}(\psi(u))-g^{\prime}\left(x_{0}\right)\right] \frac{f(\psi(u), z)}{g(\psi(u))} \mathrm{d} z \mathrm{~d} u+O\left(h^{4}+\frac{1}{n h}\right)
\end{aligned}
$$




$$
=h^{2} A_{1,2} \frac{f\left(x_{0}, y_{0}\right) g^{\prime \prime}\left(x_{0}\right)}{g^{\prime}\left(x_{0}\right)}+O\left(h^{3}+\frac{1}{n h}\right) \text {. }
$$

Now, we can calculate $E\left[\left(J_{2}^{\diamond}\right)^{2}\right]$ and $V\left[J_{2}^{\diamond}\right]$. The squared value of $J_{2}^{\diamond}$ is given by

$$
\begin{aligned}
\left(J_{2}^{\diamond}\right)^{2} & =\frac{1}{n^{4} h^{6}} \sum_{i=1}^{n} \sum_{j=1}^{n} \sum_{k=1}^{n} \sum_{m=1}^{n} K\left(\frac{Y_{i}-y_{0}}{h}\right) K\left(\frac{Y_{k}-y_{0}}{h}\right) K^{\prime}\left(\frac{G\left(X_{i}\right)-G\left(x_{0}\right)}{h}\right) \\
& \times K^{\prime}\left(\frac{G\left(X_{k}\right)-G\left(x_{0}\right)}{h}\right)\left\{\left[W\left(\frac{X_{i}-X_{j}}{h}\right)-G\left(X_{i}\right)\right]-\left[W\left(\frac{x_{0}-X_{j}}{h}\right)-G\left(x_{0}\right)\right]\right\} \\
& \times\left\{\left[W\left(\frac{X_{k}-X_{m}}{h}\right)-G\left(X_{k}\right)\right]-\left[W\left(\frac{x_{0}-X_{m}}{h}\right)-G\left(x_{0}\right)\right]\right\} \\
=: & \frac{1}{n^{4} h^{6}} \sum_{i=1}^{n} \sum_{j=1}^{n} \sum_{k=1}^{n} \sum_{m=1}^{n} \Xi^{\diamond}(i, j, k, m) .
\end{aligned}
$$

For the terms in which all $(i, j, k, m)$ are different, we can show that

$$
\begin{aligned}
& E\left[\Xi^{\diamond}(i, j, k, m)\right] \\
= & \frac{1}{h^{6}} E\left[E \left[K\left(\frac{Y_{i}-y_{0}}{h}\right) K\left(\frac{Y_{k}-y_{0}}{h}\right) K^{\prime}\left(\frac{G\left(X_{i}\right)-G\left(x_{0}\right)}{h}\right) K^{\prime}\left(\frac{G\left(X_{k}\right)-G\left(x_{0}\right)}{h}\right)\right.\right. \\
& \times\left\{\left[W\left(\frac{X_{i}-X_{j}}{h}\right)-G\left(X_{i}\right)\right]-\left[W\left(\frac{x_{0}-X_{j}}{h}\right)-G\left(x_{0}\right)\right]\right\} \\
& \left.\left.\times\left\{\left[W\left(\frac{X_{k}-X_{m}}{h}\right)-G\left(X_{k}\right)\right]-\left[W\left(\frac{x_{0}-X_{m}}{h}\right)-G\left(x_{0}\right)\right]\right\} \mid\left(X_{i}, Y_{i}\right),\left(X_{k}, Y_{k}\right)\right]\right] \\
= & \left(\frac{1}{h^{3}} E\left[K\left(\frac{Y_{i}-y_{0}}{h}\right) K^{\prime}\left(\frac{G\left(X_{i}\right)-G\left(x_{0}\right)}{h}\right)\left[\left\{h^{2} A_{1,2}\left[g^{\prime}\left(X_{i}\right)-g^{\prime}\left(x_{0}\right)\right]\right\}+O\left(h^{4}\right)\right]\right]\right)^{2} \\
= & \left(h^{2} A_{1,2} \frac{f\left(x_{0}, y_{0}\right) g^{\prime \prime}\left(x_{0}\right)}{g^{\prime}\left(x_{0}\right)}+O\left(h^{3}\right)\right)^{2} .
\end{aligned}
$$

For the terms in which $i=j$ and all $(i, k, m)$ are different,

$$
\begin{aligned}
& E\left[\Xi^{\diamond}(i, j, k, m)\right] \\
& =\frac{1}{n h^{6}} E\left[E \left[K\left(\frac{Y_{i}-y_{0}}{h}\right) K\left(\frac{Y_{k}-y_{0}}{h}\right) K^{\prime}\left(\frac{G\left(X_{i}\right)-G\left(x_{0}\right)}{h}\right) K^{\prime}\left(\frac{G\left(X_{k}\right)-G\left(x_{0}\right)}{h}\right)\right.\right. \\
& \times\left\{\left[W\left(\frac{X_{i}-X_{i}}{h}\right)-G\left(X_{i}\right)\right]-\left[W\left(\frac{x_{0}-X_{i}}{h}\right)-G\left(x_{0}\right)\right]\right\} \\
& \left.\left.\times\left\{\left[W\left(\frac{X_{k}-X_{m}}{h}\right)-G\left(X_{k}\right)\right]-\left[W\left(\frac{x_{0}-X_{m}}{h}\right)-G\left(x_{0}\right)\right]\right\} \mid\left(X_{i}, Y_{i}\right),\left(X_{k}, Y_{k}\right)\right]\right] \\
& =\frac{1}{n h^{3}} E\left[K ( \frac { Y _ { i } - y _ { 0 } } { h } ) K ^ { \prime } ( \frac { G ( X _ { i } ) - G ( x _ { 0 } ) } { h } ) \left\{\left[\frac{1}{2}-G\left(X_{i}\right)\right]\right.\right. \\
& \left.\left.-\left[W\left(\frac{x_{0}-X_{i}}{h}\right)-G\left(x_{0}\right)\right]\right\}\right] \times\left(h^{2} A_{1,2} \frac{f\left(x_{0}, y_{0}\right) g^{\prime \prime}\left(x_{0}\right)}{g^{\prime}\left(x_{0}\right)}+O\left(h^{3}+\frac{1}{n h}\right)\right) \\
& =O\left(\frac{h}{n}+\frac{1}{n^{2} h^{2}}\right) \text {, }
\end{aligned}
$$


Similarly, for the terms in which $i=k$ and all $(i, j, m)$ are different,

$$
\begin{aligned}
& E\left[\Xi^{\diamond}(i, j, k, m)\left(\frac{Y_{i}-y_{0}}{h}\right) K\left(\frac{Y_{i}-y_{0}}{h}\right) K^{\prime}\left(\frac{G\left(X_{i}\right)-G\left(x_{0}\right)}{h}\right) K^{\prime}\left(\frac{G\left(X_{i}\right)-G\left(x_{0}\right)}{h}\right)\right. \\
& \quad \times\left\{\left[W\left(\frac{X_{i}-X_{j}}{h}\right)-G\left(X_{i}\right)\right]-\left[W\left(\frac{x_{0}-X_{j}}{h}\right)-G\left(x_{0}\right)\right]\right\} \\
& \left.\left.\quad \times\left\{\left[W\left(\frac{X_{i}-X_{m}}{h}\right)-G\left(X_{i}\right)\right]-\left[W\left(\frac{x_{0}-X_{m}}{h}\right)-G\left(x_{0}\right)\right]\right\} \mid\left(X_{i}, Y_{i}\right)\right]\right] \\
& =\frac{1}{n h^{6}} E\left[K^{2}\left(\frac{Y_{i}-y_{0}}{h}\right)\left(K^{\prime}\right)^{2}\left(\frac{G\left(X_{i}\right)-G\left(x_{0}\right)}{h}\right)\left[\left\{h^{2} A_{1,2}\left[g^{\prime}\left(X_{i}\right)-g^{\prime}\left(x_{0}\right)\right]\right\}+O\left(h^{4}\right)\right]^{2}\right] \\
& =O\left(\frac{h^{2}}{n}\right) .
\end{aligned}
$$

For the terms in which $i=m$ and all $(i, j, k)$ are different,

$$
\begin{aligned}
& E\left[\Xi^{\diamond}(i, j, k, m)\right] \\
& =\frac{1}{h^{6}} E\left[E \left[K\left(\frac{Y_{i}-y_{0}}{h}\right) K\left(\frac{Y_{k}-y_{0}}{h}\right) K^{\prime}\left(\frac{G\left(X_{i}\right)-G\left(x_{0}\right)}{h}\right) K^{\prime}\left(\frac{G\left(X_{k}\right)-G\left(x_{0}\right)}{h}\right)\right.\right. \\
& \times\left\{\left[W\left(\frac{X_{i}-X_{j}}{h}\right)-G\left(X_{i}\right)\right]-\left[W\left(\frac{x_{0}-X_{j}}{h}\right)-G\left(x_{0}\right)\right]\right\} \\
& \left.\left.\times\left\{\left[W\left(\frac{X_{k}-X_{i}}{h}\right)-G\left(X_{k}\right)\right]-\left[W\left(\frac{x_{0}-X_{i}}{h}\right)-G\left(x_{0}\right)\right]\right\} \mid\left(X_{i}, Y_{i}\right)\right]\right] \\
& =O\left(\frac{h}{n}\right) \text {. }
\end{aligned}
$$

Finally, for the terms in which $j=m$ and all $(i, j, k)$ are different, we have

$$
E\left[\Xi^{\diamond}(i, j, k, m)\right]=O\left(\frac{1}{n h}\right),
$$

and

$$
V\left[J_{2}^{\diamond}\right]=O\left(\frac{1}{n h}\right) .
$$

We can see that $J_{3}^{\diamond}+J_{4}^{\diamond *}$ is asymptotically negligible. The main bias of $\widehat{f}_{Y \mid x_{0}}^{\diamond}\left(y_{0}\right)$ comes from $J_{1}+J_{2}^{\diamond}$. Since $E\left[J_{1} J_{2}\right]$ is given by

$$
\begin{aligned}
& \frac{1}{n^{3} h^{3}} \sum_{i=1}^{n} \sum_{j=1}^{n} \sum_{k=1}^{n} K\left(\frac{Y_{i}-y}{h}\right) K\left(\frac{Y_{j}-y_{0}}{h}\right) K^{\prime}\left(\frac{G\left(X_{i}\right)-G\left(x_{0}\right)}{h}\right) \\
& K^{\prime}\left(\frac{G\left(X_{j}\right)-G\left(x_{0}\right)}{h}\right)\left\{\left[W\left(\frac{X_{j}-X_{k}}{h}\right)-G\left(X_{j}\right)\right]-\left[W\left(\frac{x_{0}-X_{k}}{h}\right)-G\left(x_{0}\right)\right]\right\} \\
= & E\left[J_{1}\right] E\left[J_{2}\right]+O\left(\frac{1}{n}\right),
\end{aligned}
$$

the asymptotic variance is the same as the discrete version $\widehat{f}_{Y \mid x_{0}}^{\star}\left(y_{0}\right)$, and Theorem 2 has been proved. 
Received May 27, 2019

Revised January 10, 2020 